\title{
A Comparative Study of Simulated and Measured Gear-Flap Flow Interaction
}

\author{
Mehdi R. Khorrami* \\ NASA Langley Research Center, Hampton, Virginia, 23681 \\ Raymond E. Mineck ${ }^{\dagger}$ \\ Yorktown, Virginia, 23692 \\ and \\ Chungsheng Yao ${ }^{\ddagger}$ Luther N. Jenkins* \\ NASA Langley Research Center, Hampton, Virginia, 23681
}

The ability of two CFD solvers to accurately characterize the transient, complex, interacting flowfield associated with a realistic gear-flap configuration is assessed via comparison of simulated flow with experimental measurements. The simulated results, obtained with NASA's FUN3D and Exa's PowerFLOW ${ }^{\circledR}$ for a highfidelity, $18 \%$ scale semi-span model of a Gulfstream aircraft in landing configuration $\left(39^{\circ}\right.$ flap deflection, main landing gear on and off) are compared to two-dimensional and stereo particle image velocimetry measurements taken within the gear-flap flow interaction region during wind tunnel tests of the model. As part of the benchmarking process, direct comparisons of the mean and fluctuating velocity fields are presented in the form of planar contour plots and extracted line profiles at measurement planes in various orientations stationed in the main gear wake. The measurement planes in the vicinity of the flap side edge and downstream of the flap trailing edge are used to highlight the effects of gear presence on tip vortex development and the ability of the computational tools to accurately capture such effects. The present study indicates that both computed datasets contain enough detail to construct a relatively accurate depiction of gear-flap flow interaction. Such a finding increases confidence in using the simulated volumetric flow solutions to examine the behavior of pertinent aerodynamic mechanisms within the gear-flap interaction zone.

\section{Nomenclature}

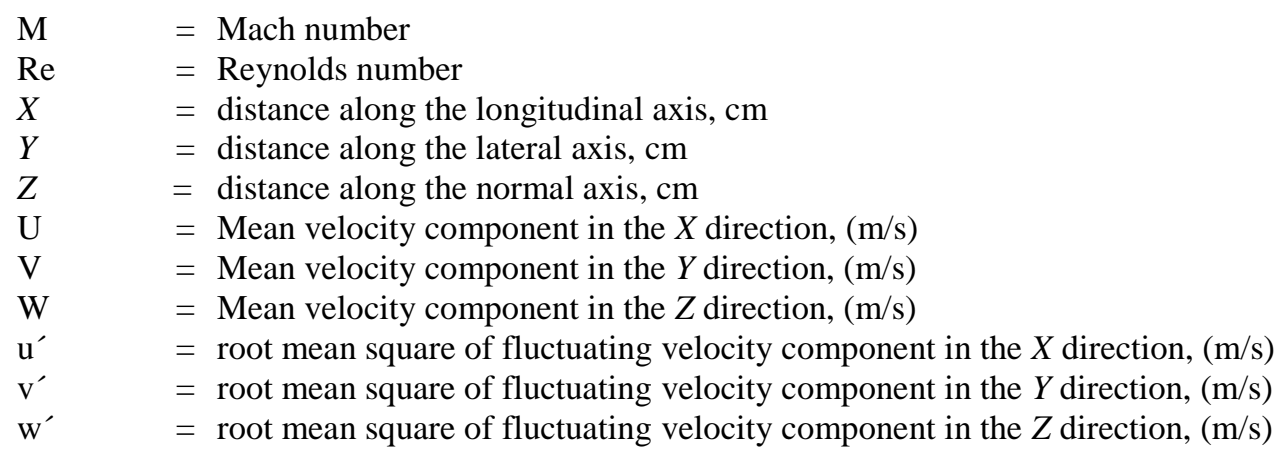

\section{Introduction}

A significant portion of aircraft noise during landing is generated by the airframe. Because of their importance to community noise near airports, major airframe noise sources such as the landing gear and wing high-lift devices (wing

\footnotetext{
* Aerospace Engineer, Computational AeroSciences Branch, Associate Fellow AIAA.

${ }^{\dagger}$ Consultant.

* Aerospace Engineer, Flow Physics and Control Branch.
} 
leading-edge slat and trailing-edge flaps) have received considerable attention during the past two decades. ${ }^{1}$ In isolation, these noise sources have been the subject of numerous experimental and computational studies, as summarized in Ref. 1. However, much less is known about component interaction (also called installation) effects that arise when the presence of an airframe component may positively or negatively impact the local flow, and thus the noise generated by a nearby component. Gear-flap interaction for a realistic geometry in a relevant aerodynamic environment is among the installation effects that have been virtually unexplored.

An accurate representation of the local flow interactions that occur when airframe components such as landing gear and flaps are deployed in proximity of each other and the corresponding flow features responsible for noise generation via computational simulations is a very challenging task. Important issues such as 1) correct characterization of the flow field associated with the numerous components of a complex landing gear, including the finer features known as gear dressing; 2) integrity of the gear turbulent wake, as it contains a broad range of spatio-temporal flow features, and 3) consistent wake convection over relatively large distances to ensure proper interaction with the flap tip region, must be considered.

In this paper, we present a direct comparison between simulated and measured off-surface mean and fluctuating velocity fields of a gear-flap interaction zone for a realistic setup. The simulated configuration is that of a high fidelity, $18 \%$ scale semi-span Gulfstream aircraft model in landing configuration with flap deflected at $39^{\circ}$ with and without the main landing gear. The two independent computational datasets used in this comparison are the same as those previously used to benchmark the accuracy of predicted steady and unsteady surface pressure fields for the same geometry. ${ }^{2-3}$ Time-dependent solutions of the flowfield associated with the model were generated with an in-house unstructured compressible Navier-Stokes solver and lattice Boltzmann based solver.

The corresponding experimental dataset employed in this paper was acquired using particle image velocimetry (PIV) to measure instantaneous velocities in the immediate vicinity of the main landing gear and its wake and near the inboard tip of the flap. These measurements were performed during the third entry of a series of aeroacoustic tests conducted in the NASA Langley Research Center (LaRC) 14- by 22-Foot Subsonic Tunnel (14x22) using the 18\% scale model in landing configuration as the test bed. ${ }^{4-7}$

\section{Dataset Description}

The computational and experimental datasets under consideration were obtained using an $18 \%$ scale, semi-span, high-fidelity reproduction model of a Gulfstream aircraft (see Fig. 1). The geometry comprises a fuselage, wing, flap, flow-through nacelle, pylon, and main landing gear. A full description of this model is provided in Ref. 4. The majority of the off-body PIV measurements were obtained at a freestream Mach (M) number of 0.2, angle of attack (AOA) of $3^{\circ}$, and flap deflection angle of $39^{\circ}$ with the landing gear on. The corresponding Reynolds number $(\mathrm{Re})$ based on the mean aerodynamic chord of the model wing was $3.40 \times 10^{6}$. In addition, a limited amount of data was acquired with the landing gear off. A detailed account of the model setup and flow measurements in the 14x22 tunnel for the PIV test campaign is given in Ref. 7.

\section{A. Computational Datasets}

The numerical datasets employed in this study were obtained using two distinct simulation methodologies. For one set, NASA's FUN3D ${ }^{8}$ unstructured compressible Navier-Stokes solver was used to simulate the unsteady flow field associated with the aircraft model. To capture the more prominent flow structures, detached eddy simulation (DES) in conjunction with the one-equation Spalart-Allmaras turbulence model ${ }^{9}$ was used for the calculations. The computed solutions were obtained for a representation of the open-jet $14 \times 22$ tunnel configuration (i.e., no slip wall boundary condition on the floor and free-air boundary condition in all directions above the floor), maintaining the same test conditions and model configurations as in the PIV setup. The two mixed-element grids used in the FUN3D computations, comprised of $243.9 \times 10^{6}$ and $232.9 \times 10^{6}$ nodes, corresponded to the baseline configurations of flap $39^{\circ}$ with the main gear on and off, respectively. A sample planar cut of the mesh in the gear-flap region is shown in Fig. 2. Scrutiny of the grid at several random locations within the gear-flap interaction zone indicated that the majority of the cell sizes fell within the range $0.6 \mathrm{~mm}$ to $2.0 \mathrm{~mm}$. For more details on the FUN3D computations, the reader is directed to Ref. 2.

For the second computational dataset, Exa Corporation's lattice Boltzmann PowerFLOW ${ }^{\circledR 10}$ solver was used to conduct the time-dependent simulations for the same setup as in the FUN3D case. The lattice Boltzmann equations represent an alternative mesoscopic formulation to the classical macroscopic Navier-Stokes equations describing a compressible unsteady flow of a continuum. For high Reynolds number flows, such as those addressed in this work, turbulence modeling is incorporated into the lattice Boltzmann model by replacing the relaxation time, which is related 
to the thermodynamic chaotic motion of fluid particles, by a turbulent relaxation time that models the effect of turbulent chaotic motion on the statistics of fluid particle collisions. The turbulent kinetic energy and the turbulent dissipation are obtained by solving a variant of the RNG k- $\varepsilon$ model. ${ }^{11}$ In this respect, the turbulent lattice Boltzmann flow model is analogous to a hybrid URANS-LES formulation. Extremely fine resolutions were maintained in regions adjacent to the flap tips, surrounding the main landing gear, and gear-flap interaction zone. The grid generated for the configuration with the main gear on had a total of $2.9 \times 10^{9}$ Cartesian volume elements (voxels). Sample planar cuts of the grid within the gear-flap interaction region are shown in Fig. 3. For the finest resolution used, examination of the grid within the gear-flap interaction zone indicated that the majority of the voxel sizes are in the range of $0.5 \mathrm{~mm}$ to $1.5 \mathrm{~mm}$. The corresponding grid for the configuration without the main gear was comprised of $2.6 \times 10^{9}$ voxels. A complete description of the PowerFLOW® simulations is provided in Ref. 3.

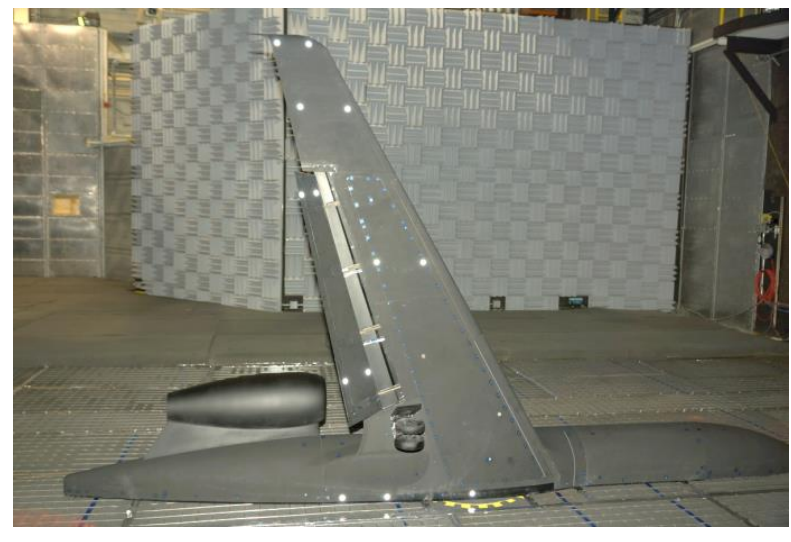

a) Model installed in NASA LaRC 14x22 tunnel

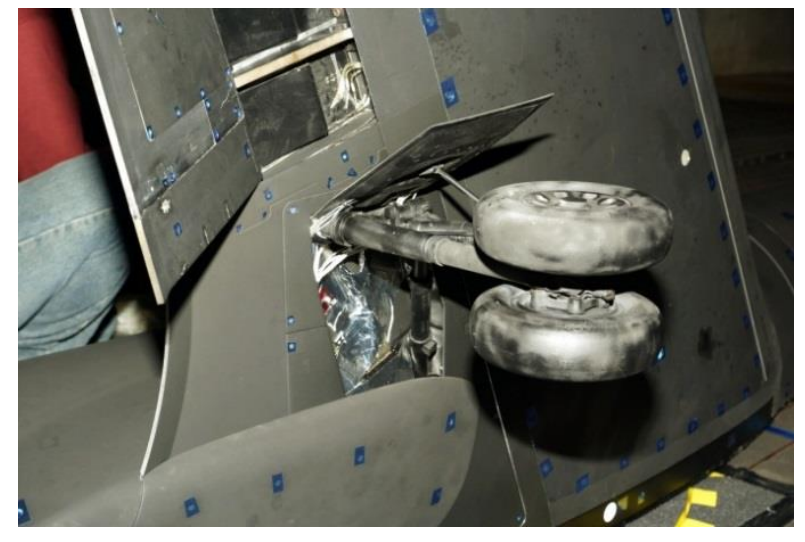

b) Close-up view of gear-flap region

Figure 1. 18\% scale semi-span model of a Gulfstream aircraft in 14x22 tunnel

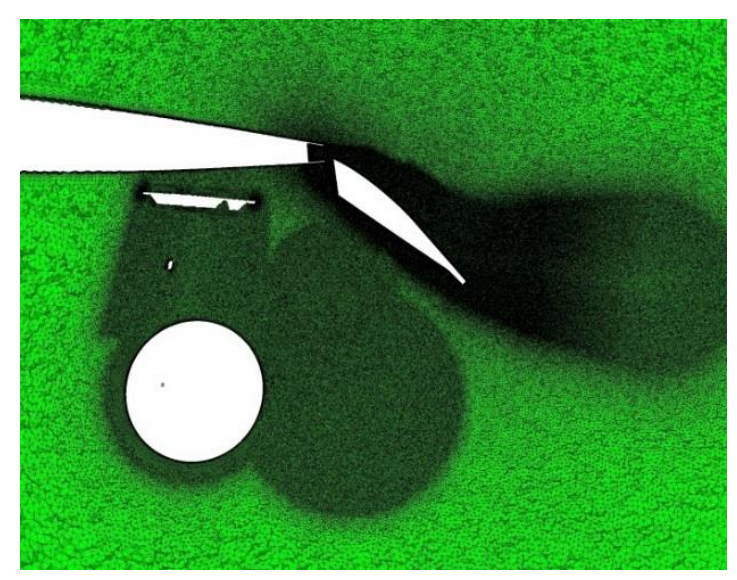

a) Planar cut bisecting one of the wheels

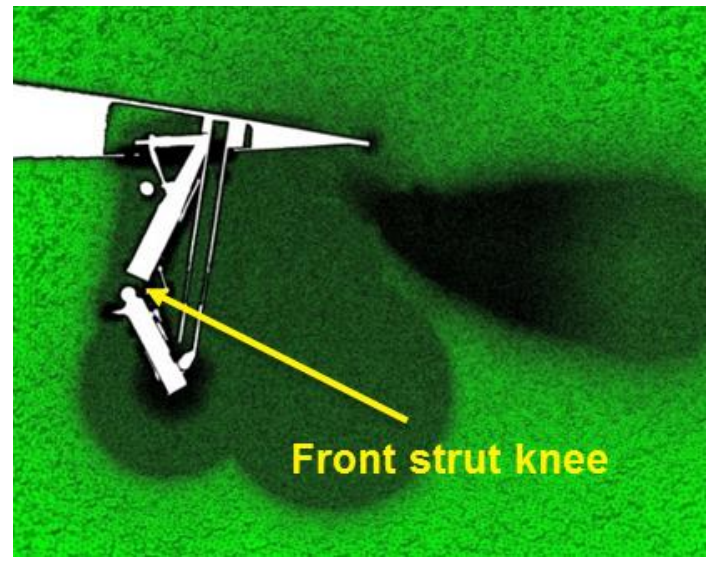

b) Planar cut bisecting gear

Figure 2. Close-up views of FUN3D unstructured mesh in the gear-flap interaction zone 

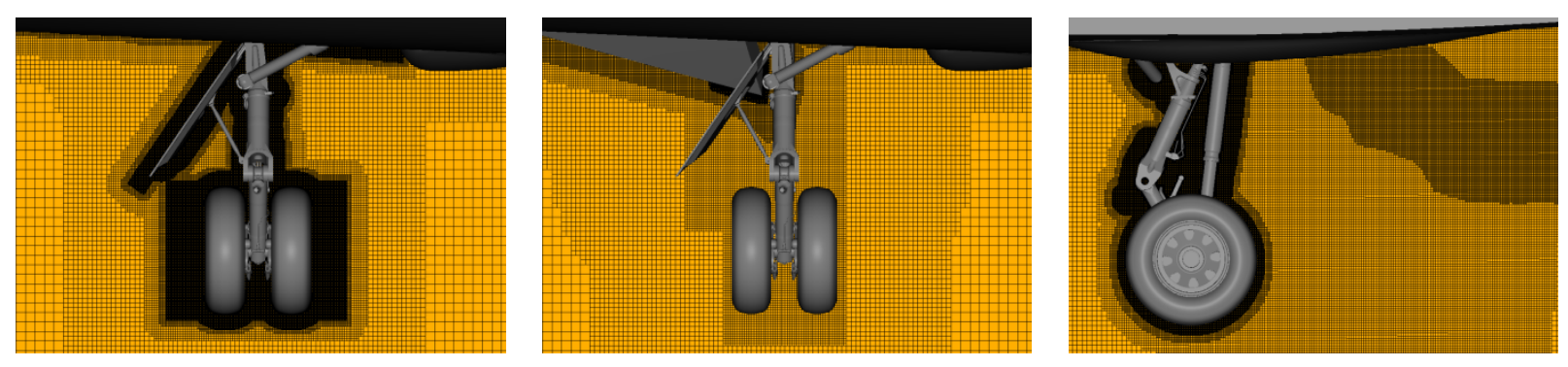

Figure 3. Close-up views of the PowerFLOW® mesh surrounding the gear (every second line is shown)

\section{B. Experimental Dataset}

Extensive PIV measurements of the $18 \%$ scale semi-span model with a focus on mapping the flowfield in the landing gear wake and within the gear-flap interaction region were conducted in the $14 \times 22$ tunnel during April of 2013 (Fig. 4). Two different PIV configurations were used to acquire data in the wake of the landing gear. The first configuration consisted of a two-dimensional (2D) PIV system to measure two components of velocity in X-Y planes behind the landing gear. The field of view (FOV) for this PIV configuration was approximately $300 \times 200 \mathrm{~mm}$ (width $\mathrm{x}$ height) and the working distance was approximately 4.27 meters ( 14 feet) from the underside of the model. The location and orientation of the measurement planes relative to the landing gear are shown in Fig. 5. The second PIV configuration consisted of a stereo PIV (SPIV) system that was used to measure three components of velocity in crossflow planes behind the landing gear. The SPIV cameras were arranged on opposite sides of the light sheet as it was projected to the measurement areas through the windows in the tunnel floor. The location and orientation of the individual measurement planes for the second SPIV configuration are shown in Figs. 6a and 6b. The actual measurement area for the two PIV configurations was smaller than the specified FOVs because of constraints on where the laser sheet could be projected through the tunnel floor, flare caused by impingement of laser light on the model, and acute blockage of the measurement area in certain camera views by the complicated geometry of the landing gear/flap. A detailed account of the PIV apparatus and test campaign is provided in Ref. 7.

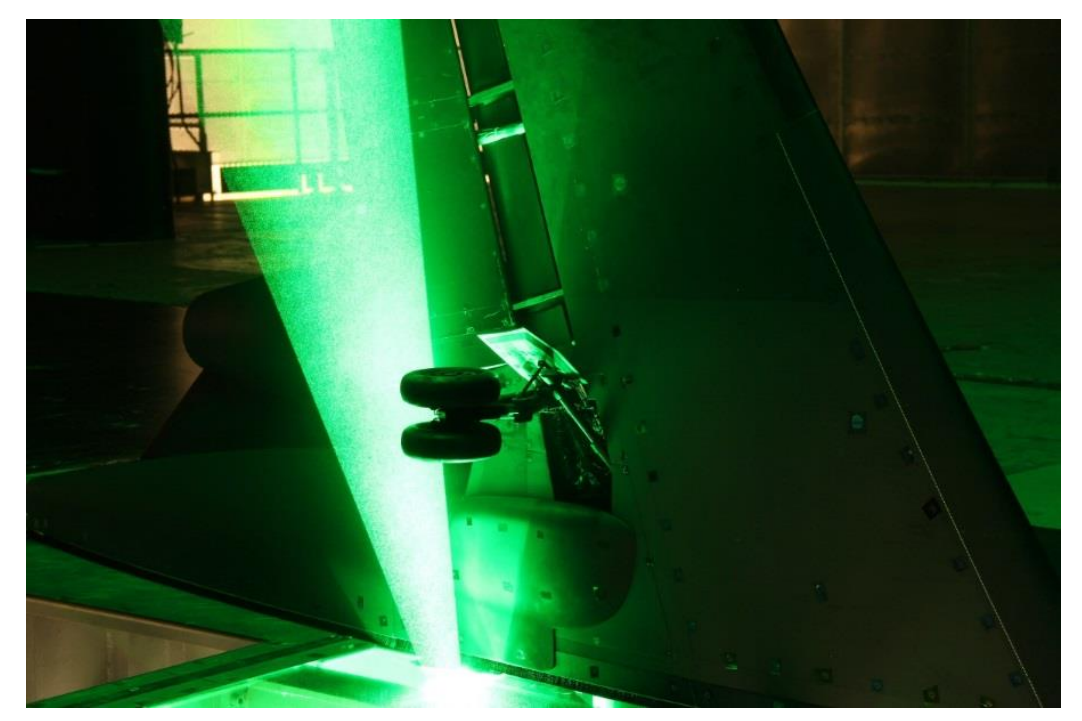

Figure 4. $18 \%$ scale semi-span model in $14 \times 22$ tunnel during PIV test campaign 


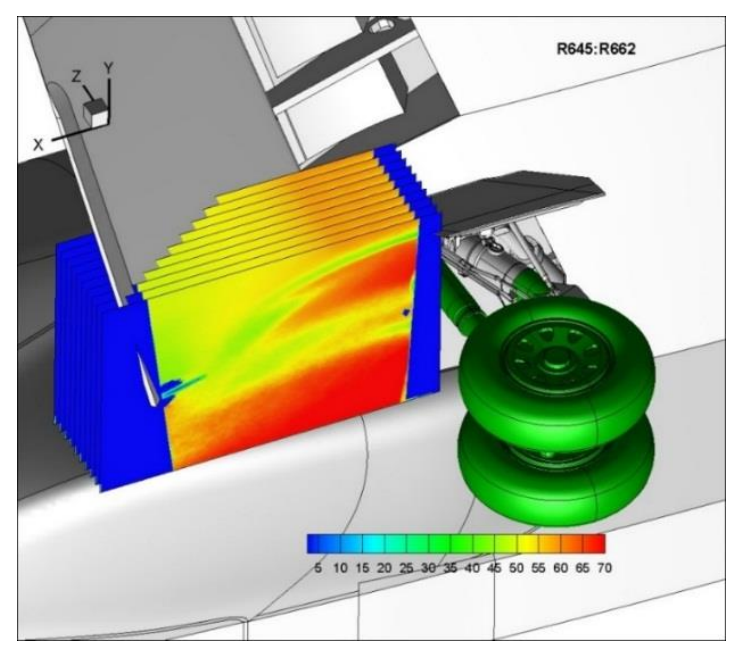

Figure 5. Location and orientation of 2D-PIV measurement planes

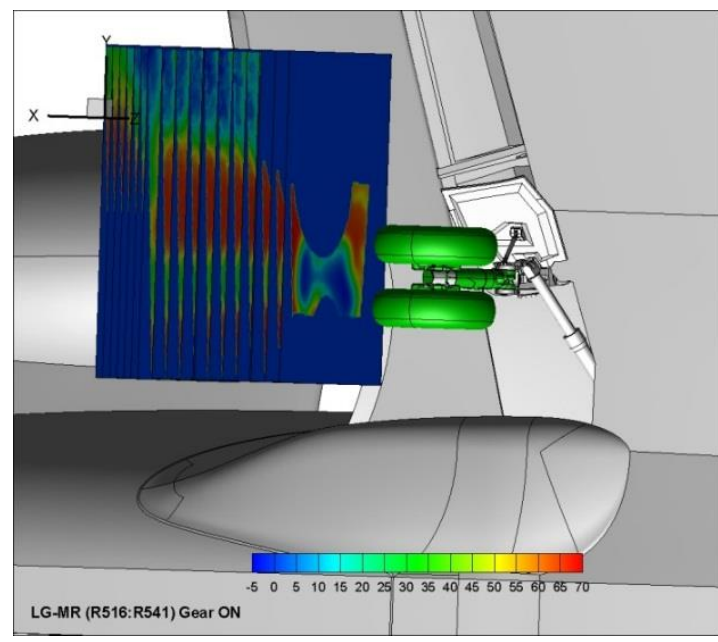

a) Wake associated with wheel and lower portion of strut

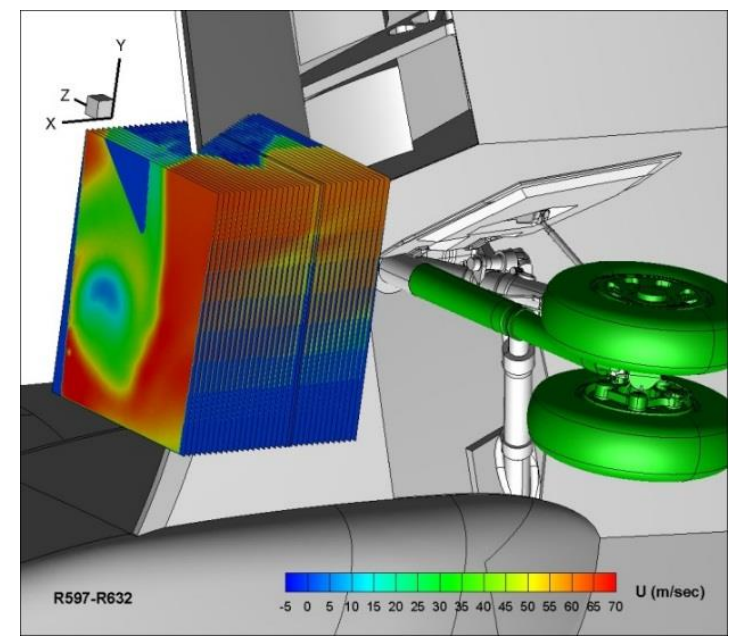

b) Upper portion of strut/inboard edge of flap

Figure 6. Location and orientation of SPIV measurement planes

As in the case of any computational simulation, the quality of the PIV data depends on the spatial resolution of the measurement planes. In particular, given the multitude of high gradient flow regions encountered within the gear-flap interaction zone, it is necessary to acquire a better understanding of how accurately these regions have been resolved. Furthermore, the complex PIV setup and test configurations/conditions introduced an additional source of measurement uncertainty in the collected data. The effects of these two sources of uncertainty are examined briefly in the following sections.

\section{a. Spatial Resolution Effects}

Given the large number of measurement planes and the limited tunnel time available, acquiring PIV measurements at multiple spatial resolutions was confined to a few select planes. One of these SPIV planes is shown in Fig. 7. As expected, the plane identified as medium resolution (Run 537) having a $4.90 \mathrm{~mm}$ by $4.90 \mathrm{~mm}$ spatial resolution in $\mathrm{Y}$ and $\mathrm{X}$ coordinates encompasses a much larger FOV that contains the wake generated by the wheels and lower portion of the gear strut. In contrast, the better resolved plane referred to as high resolution (Run 580) having a resolution of $1.81 \mathrm{~mm}$ by $1.81 \mathrm{~mm}$ in both coordinates is confined to a small portion of the plane with lower resolution. In Fig. 7a, the three components of the mean velocity are shown. The upper row in the figure shows the contours obtained at higher resolution and the lower row displays contours acquired with our nominal (medium) resolution. Because of the differences in setup and masking, notice that the upper edge of the contours for medium and high resolution data have different shapes, with the medium resolution covering a larger area. Regardless of the setup, the quality of the PIV 
data degrades noticeably in the periphery of the scanned area or whenever there is interference from light reflected by the model surfaces. Corresponding contours for the root-mean-square (rms) values of the fluctuating field are shown in Fig. 7b. Overall, good qualitative agreement for the global features of the mean and fluctuating velocity fields is observed between the two resolutions. It is worth noting that the medium and high resolution data sets were acquired several days apart.
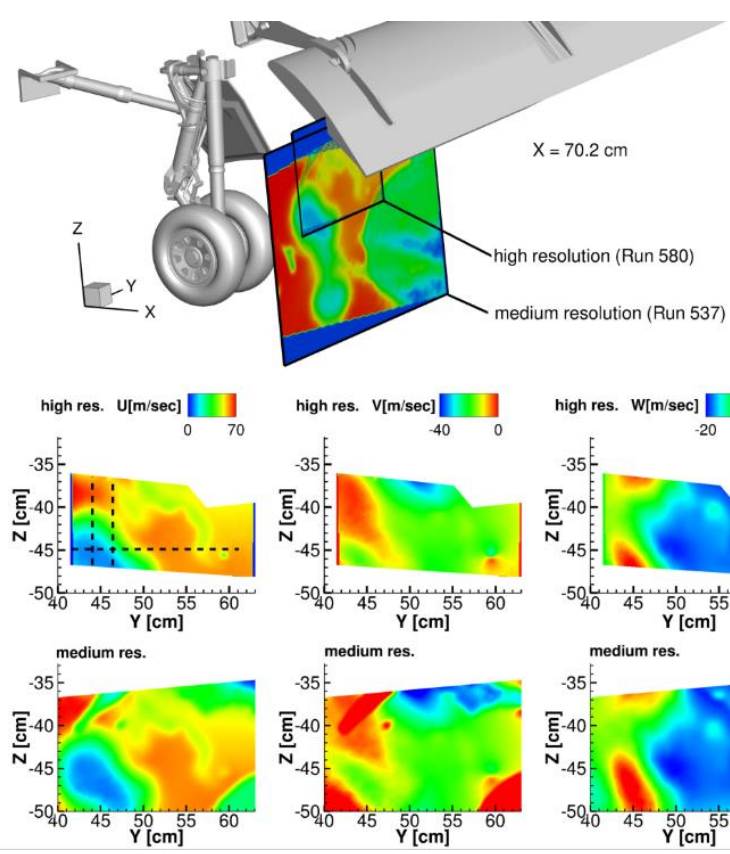

$\mathrm{U}$

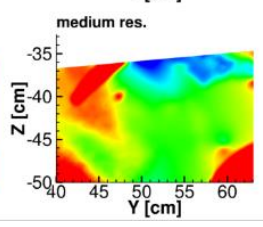

$\mathrm{V}$
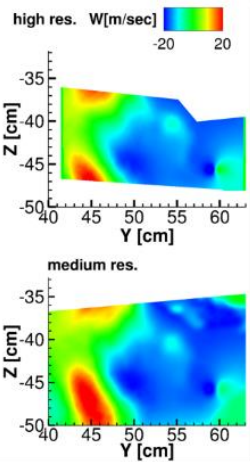

$\mathrm{W}$
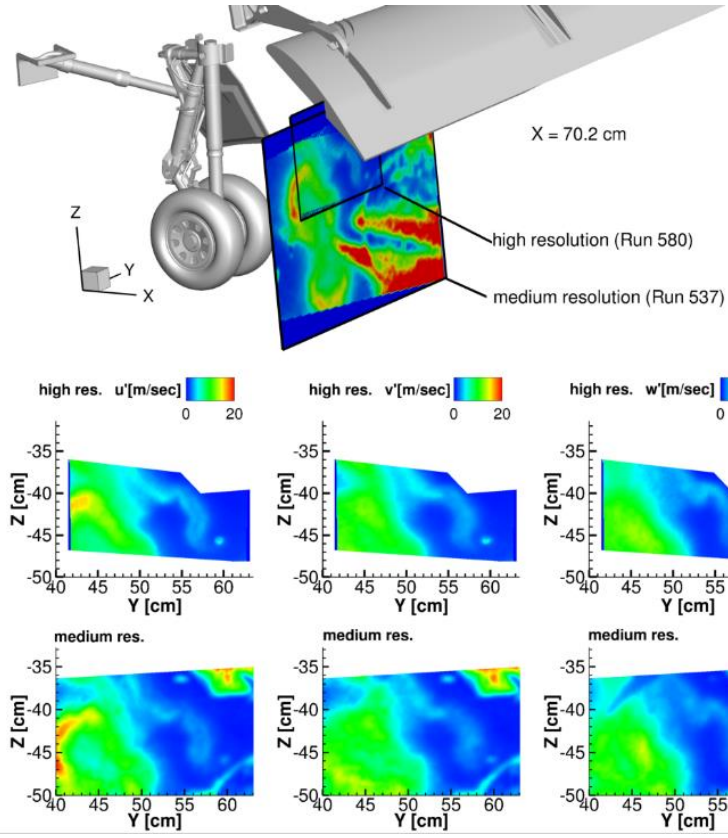

$u^{\prime}$
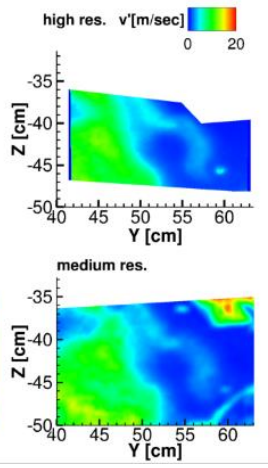

$\mathrm{V}^{\prime}$
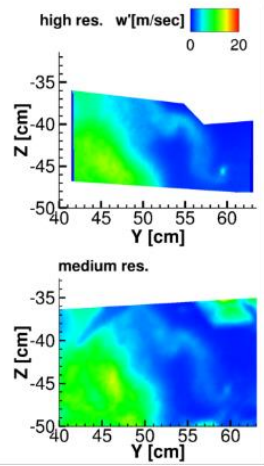

$\mathrm{w}^{\prime}$

a) Mean velocity

b) Fluctuating velocity

Figure 7. Spatial resolution effects on measured velocity field $(X=70.2 \mathrm{~cm})$

Quantitative comparisons between medium and high resolution data sets are shown in Fig. 8. The mean and fluctuating velocity profiles along the $\mathrm{Y}$ coordinate at $\mathrm{X}=70.2 \mathrm{~cm}$ and $\mathrm{Z}=-45.0 \mathrm{~cm}$ (dashed horizontal line in the highresolution contour of Fig. 7a) are plotted in Fig. 8a. Good agreement between the two sets of profiles is observed, demonstrating that the lower resolution dataset provides sufficient spatial detail to capture all the relevant features of the flow field. The velocity profiles along the Z-coordinate at $\mathrm{X}=70.2 \mathrm{~cm}$ and $\mathrm{Y}=44.0 \mathrm{~cm}$ (leftmost dashed vertical line in Fig. 7a) are plotted in Fig. 8b. Similar to the mean velocities of Fig. 8a, there is good agreement between the two resolutions. The largest difference occurs in the vertical component of the mean and fluctuating velocity (W and $\left.\mathrm{w}^{\prime}\right)$ fields. At most measurement locations, the mean (U, V, W) and standard deviation $\left(\mathrm{u}^{\prime}, \mathrm{v}^{\prime}, \mathrm{w}^{\prime}\right)$ of the velocity were computed using 600 to 1000 PIV images, which were deemed sufficient to estimate turbulence quantities and examine the level of flow unsteadiness (see Ref. 7 for details). In general, the first order moment based on 1000 PIV samples follows nearly a normal distribution. Gaussian statistics estimate that the uncertainty at $95 \%$ confidence in the mean is within $\pm 1.96 \sigma / \sqrt{ } 1000$, where $\sigma$ is the estimated standard deviation. Higher order velocity moments require a substantially larger number of samples to approach a normal distribution. Intrinsically, PIV measurement itself has limited dynamic range due to finite pixel resolution. Therefore, the uncertainty estimation for higher order moments is not addressable here. System bias errors for both SPIV and PIV measurements in this test are not available. Samples of the estimated levels of uncertainty in the mean quantities are given in Fig. 8. In addition to data uncertainty, some of the observed differences could be caused by the day-to-day experiment and measurement variability, as will be discussed in the next section. 

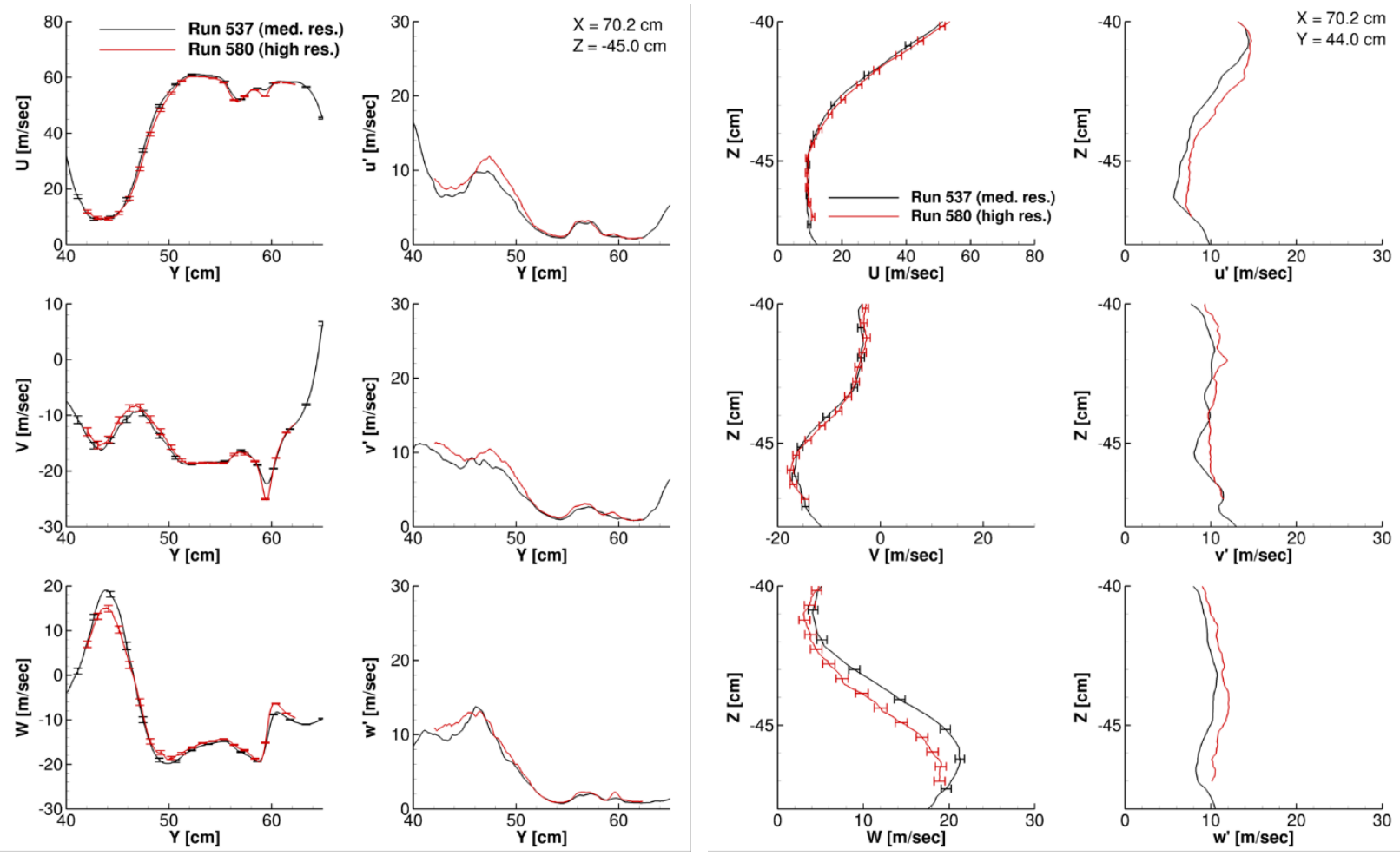

a) Y profiles

b) $\mathrm{Z}$ profiles

Figure 8. Extracted velocity profiles along $Y$ and $Z$ coordinates

\section{b. Measured Data Repeatability}

Due to PIV operational intricacy, measurement of a particular flow region may extend over multiple days. Thus, it was necessary to explore day-to-day variability of the measured data by repeating data collection for a few configurations. Within the bounds of the allocated tunnel time, this ad-hoc approach was deemed sufficient to provide useful information on data repeatability. A rigorous assessment of the various sources of PIV data variability, such as a) error in setting the model AOA, b) stability of the optic system, and c) daily variation in the tunnel test conditions due to changes in external weather, was beyond the scope of the present study.

Sample contour plots of the $U$ and $u^{\prime}$ velocity components at two streamwise locations of $X=70.2 \mathrm{~cm}$ (Runs 580, 583, and 597) and $84.2 \mathrm{~cm}$ (Runs 522, 523, and 529) are shown in Fig. 9. These runs were executed over multiple days and AOA changes, which necessitated recalibrating the PIV system each time. Note that the boundaries of the areas shown in the contour plots differ for some of the runs. This is caused by application of slightly different masking during data processing. Inspection of the contour plots in Fig. 9 reveals that good data repeatability was attained, producing flow features that are very similar for all three runs. Although not shown, similarly close agreement for the other velocity components ( $\mathrm{V}, \mathrm{v}^{\prime}, \mathrm{W}$, and $\left.\mathrm{w}^{\prime}\right)$ was also observed. A quantitative assessment of the day-to-day differences in the measurements can be obtained by examining velocity profiles along a fixed coordinate. Sample profiles for all three components along $\mathrm{Y}$ and $\mathrm{Z}$ coordinates at $\mathrm{X}=70.2 \mathrm{~cm}$ station are shown in Fig. 10. Corresponding plots at $X=84.2 \mathrm{~cm}$ are displayed in Fig. 11. Despite complexities of the PIV setup and measurement process, good data repeatability is observed. Nevertheless, noticeable differences show up. In general, these differences are more pronounced at the upstream $(X=70.2 \mathrm{~cm})$ station, typically between Run 580 and the other two Runs (583 and 597). The largest differences are observed for the V component (Fig. 10a) and the $\mathrm{w}^{\prime}$ component (Fig. 10b). As mentioned in the previous section, the magnitude of these differences is generally on par with the spatial resolution effects displayed in Figs. 8a and 8b. It is inherently difficult to isolate the attributed sources from the overall differences under the constraints of this test. 

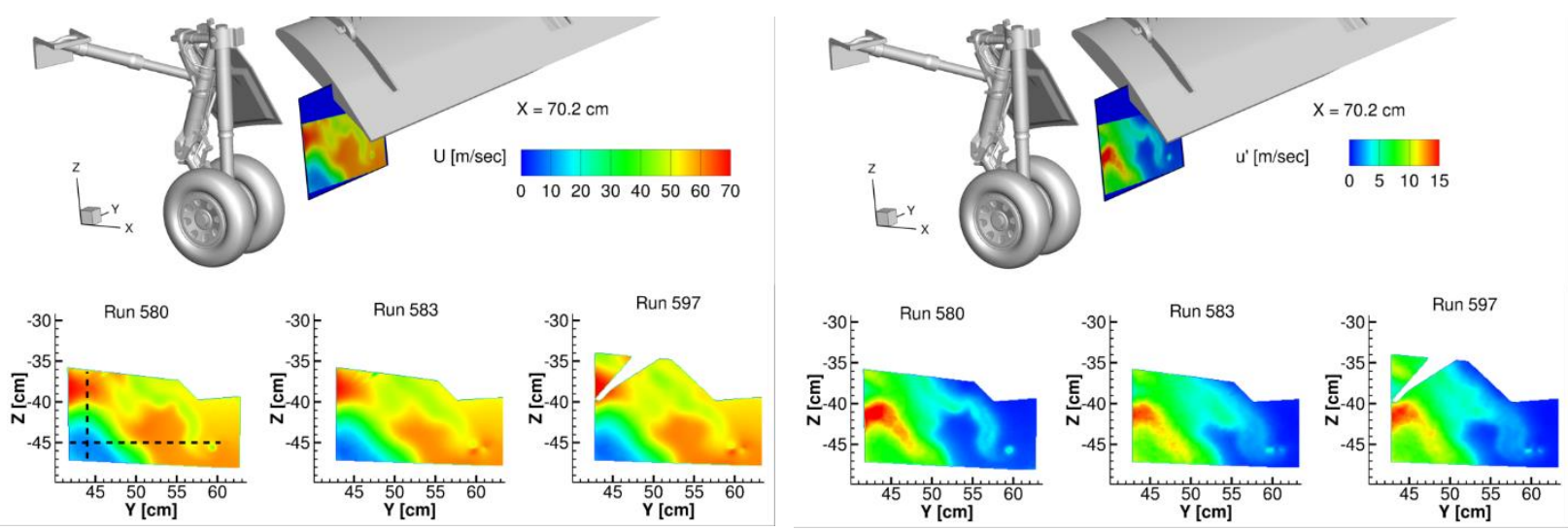

a) U velocity field at $X=70.2 \mathrm{~cm}$ plane

b) $u^{\prime}$ velocity field at $X=70.2 \mathrm{~cm}$ plane
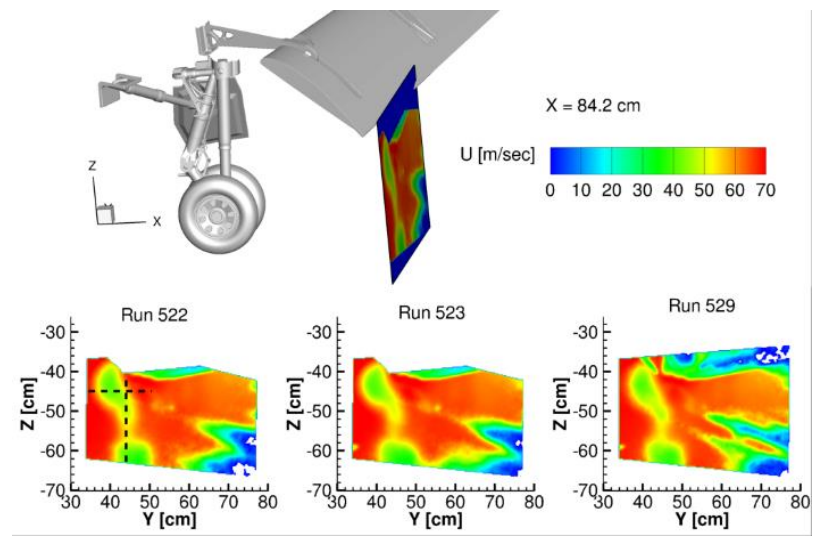

c) U velocity field at $X=84.2 \mathrm{~cm}$ plane
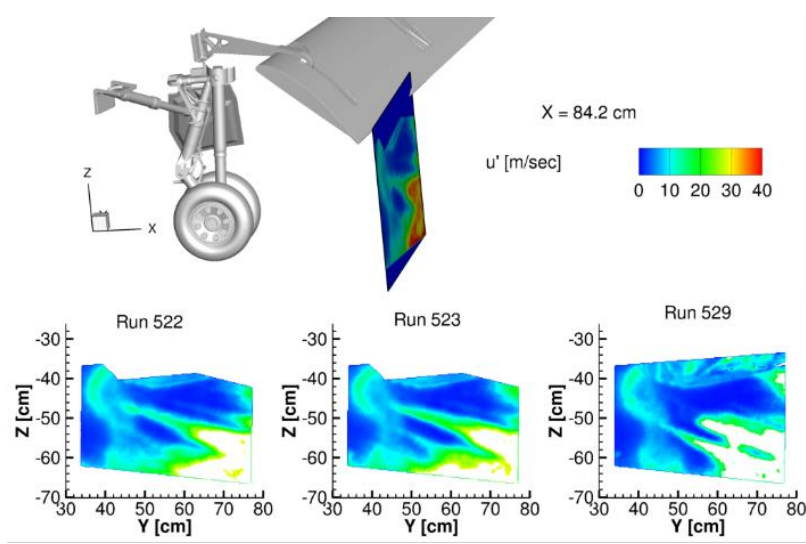

d) $u^{\prime}$ velocity field at $X=84.2 \mathrm{~cm}$ plane

Figure 9. Measured mean and fluctuating streamwise velocity contours from runs acquired at different days. 

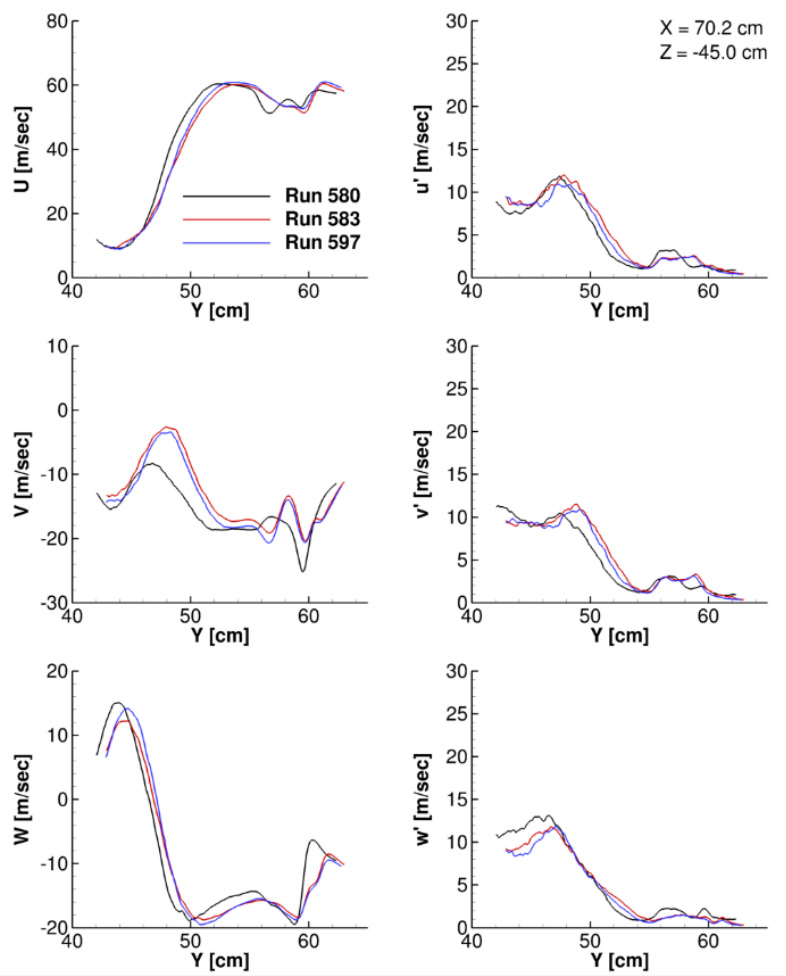

a) Y profiles
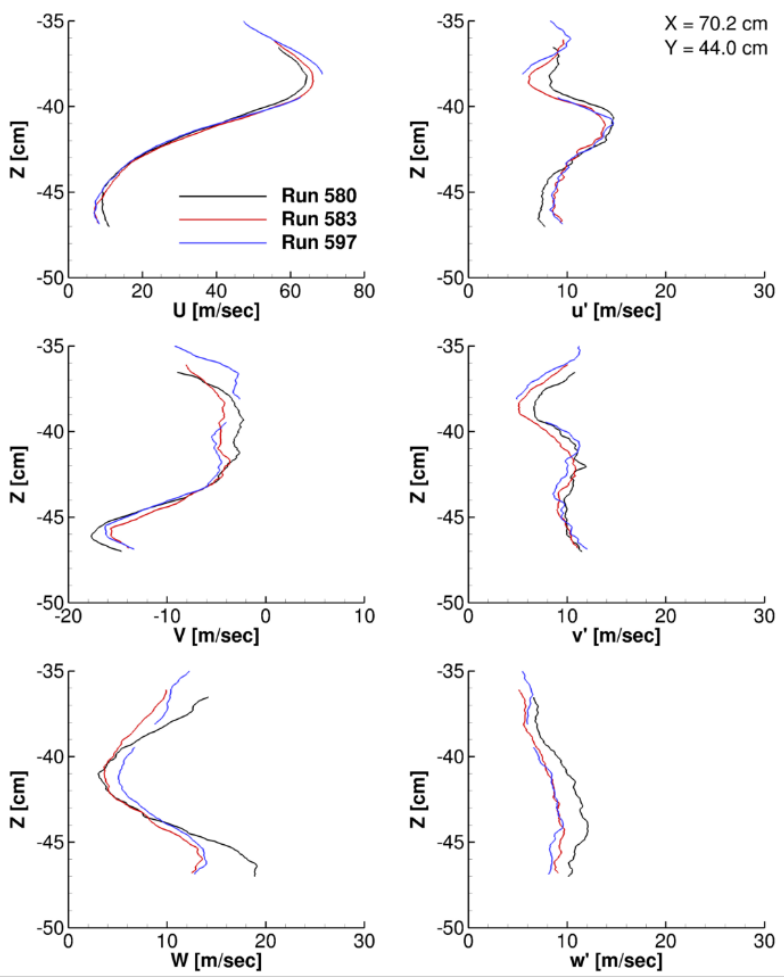

b) Z profiles

Figure 10. Mean and fluctuating velocity profiles from runs acquired at different days $(X=70.2 \mathrm{~cm})$.
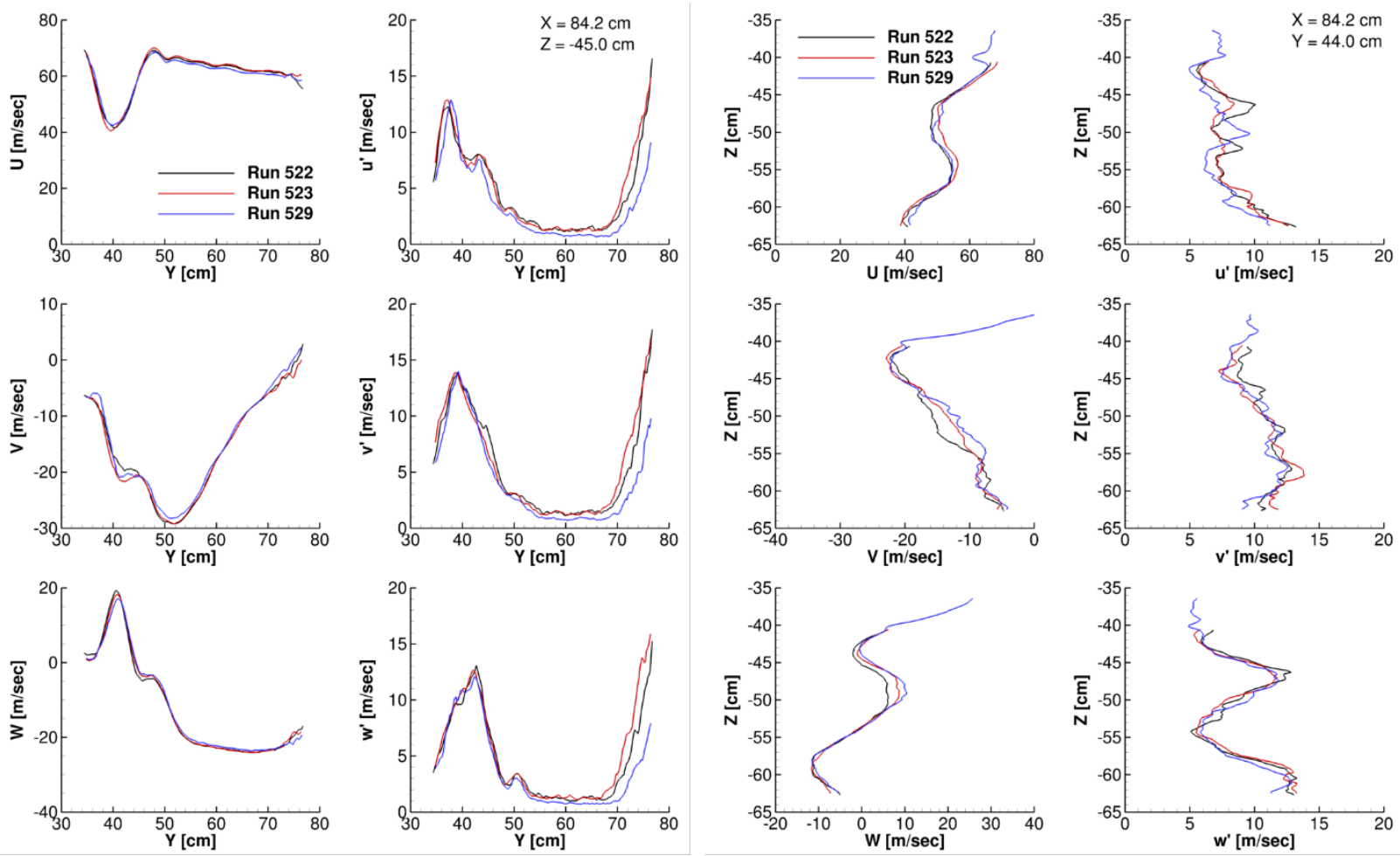

a) Y profiles

b) $\mathrm{Z}$ profiles

Figure 11. Mean and fluctuating velocity profiles from runs acquired at different days $(X=84.2 \mathrm{~cm})$. 


\section{Results and Discussion}

The large amount of velocity data from numerous 2D-PIV and SPIV measurement planes precludes inclusion of each individual plane in the present study. To demonstrate the observed general trends, sample comparison plots of the mean and root-mean-square (rms) fluctuation velocities at a select number of planes obtained for the two simulated flowfields and those of the PIV measurements are presented in this section.

\section{A. Spatial Resolution Effects on Simulated Flowfield}

As was the case for the PIV measurements, spatial resolution is a critical factor affecting the accuracy of the simulated flowfield. Because the mesh generation process employed by the PowerFLOW@ solver is octree based, it was relatively simple to conduct the simulation on grids with increasing resolution. Generation of the unstructured meshes employed by the FUN3D solver is a tedious and complex process that forced us to focus on creating a single grid of the finest resolution that would fit within the available computational resources and project schedule. Therefore, to highlight the effect of spatial resolution on the accuracy of the computed flowfield, we restrict ourselves to comparison of the medium and fine resolution results obtained with PowerFLOW®. The spatial refinement is global, with cell size in the fine grid being 1/1.5 times smaller than the corresponding medium grid cell size. For completeness, corresponding PIV measurements are also included.

The mean and fluctuating streamwise velocity ( $\mathrm{U}$ and $\mathrm{u}^{\prime}$ ) contours capturing the wake of the main landing gear at $\mathrm{X}=68.2 \mathrm{~cm}$ are plotted in Figs. 12a and 12b. Very good agreement between the medium and fine resolution CFD results was attained. Most of the main flow features observed in the PIV contours are closely captured by the simulations even at medium resolution. Grid dependency effects are clear in the sample velocity profiles extracted along the $\mathrm{Y}$ coordinate at $\mathrm{Z}=-45.0 \mathrm{~cm}$. Note that these effects are minor, indicating that the simulated velocities are well converged, with the finer resolution profiles being in better agreement with the PIV profiles. The corresponding contours for the $\mathrm{W}$ and $\mathrm{w}^{\prime}$ components are shown in Figs. $12 \mathrm{c}$ and $12 \mathrm{~d}$. In these figures, the $\mathrm{Y}$ profiles were extracted at $\mathrm{Z}=$ $60.0 \mathrm{~cm}$. The computed contours are remarkably well converged and both resolutions produce results that are in good agreement with the measurements, except for a somewhat elongated main lobe where the PIV peak positive W values occur. The $\mathrm{w}^{\prime}$ profiles shown in Fig. 12d indicate that good solution convergence was achieved, with the peak rms fluctuation levels being about 20\% larger than the measured amplitudes. As is the case with many other measurement techniques, larger differences are to be expected in the higher moment velocities. Nonetheless, given the excellent agreement observed for the mean W velocity (Fig. 12c), the difference in the amplitude of $\mathrm{w}^{\prime}$ is somewhat disappointing. For the PIV measurements reported here, the sources and magnitude of data uncertainty depend on a variety of factors that make error quantification a difficult task. Although not shown here, acceptable grid convergence was observed at most other locations/regions where PIV measurements were acquired, thus making the fine resolution PowerFLOW® dataset our best available solution.

Despite the good agreement shown between simulated and measured data (Fig. 12), unusual trends/behavior were observed occasionally in various regions of the gear-flap zone. To highlight some peculiarities of the PIV measurements, $\mathrm{W}$ and $\mathrm{w}^{\prime}$ profiles extracted along the $\mathrm{Y}$ coordinate at $\mathrm{Z}=-45.0 \mathrm{~cm}$, are shown in Fig. 13. Note from Figure 13a that the sampling location cuts through the elongated flow structure mentioned earlier; thus, we expect this cut to capture the largest difference between the computed and measured $\mathrm{W}$ velocities. While the CFD solutions indicate a reasonably converged result, not surprisingly, there are significant differences in the peak W magnitude with measured levels even though the profile shapes are somewhat similar (Fig. 13a). Intuitively, the higher velocity gradients observed in the measured $\mathrm{W}$ component suggest that one should expect higher fluctuation levels in the $\mathrm{w}^{\prime}$ component. As seen in Fig. 13b, this is not the case. Indeed, there is very good agreement among computed and measured rms profiles with the latter actually producing lower amplitudes.

Further evidence of these unusual trends between measured and computed velocity fields is given in Figs. 14 and 15. In Fig. 14, contours of the mean and fluctuating components of streamwise velocity for the gear deployed configuration downstream of the flap trailing edge, capturing the tip vortex, are displayed. The simulated contours for $\mathrm{U}$ (Fig. 14a) show a grid-converged field that is in very good agreement with the measurements. Notice that the vortex core has a large velocity deficit, exhibiting wake-like behavior. The magnitude of this deficit is clearly shown in the extracted mean velocity profiles along the $\mathrm{Y}$ coordinate at $\mathrm{Z}=-36.5 \mathrm{~cm}$. Again, the CFD profiles are in a reasonable agreement with each other. Note from the mean velocity profiles that there is an apparent Y-shift in of about two centimeters in the computed vortex position relative to the measured position. This shift can be attributed to several factors, foremost among them the differences between computational (free-air) and experimental (open-jet) setups and the presence of a slight flow angularity in the tunnel incoming flow. The $\mathrm{u}^{\prime}$ component of the fluctuating velocity is shown in Fig. 14b. The medium and fine resolution contours indicate that a fairly well converged field was achieved, while comparison with the measured contours reveals that the computed field contains larger amplitude fluctuations. A quantitative assessment of the differences in fluctuation amplitudes can be obtained from the $u^{\prime}$ profiles extracted 
along the Y-coordinate. Although the PIV profile has the same shape (character) as the simulated profiles, measured fluctuation amplitudes are generally 20\%-30\% lower. The same shift in vortex position observed in Fig. 14a is also observed here.

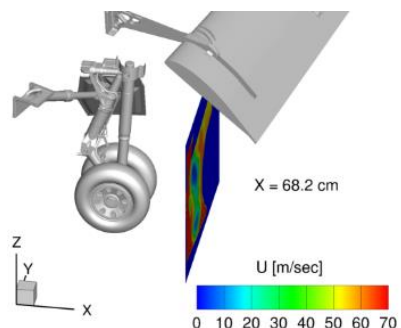

$\begin{array}{rrrr}0 & 10203040506070 \\ \text { PIV } & \quad \text { PowerFLOW - fine }\end{array}$
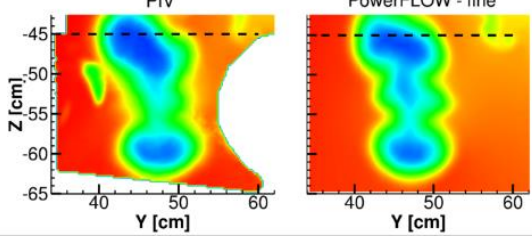

a) Mean U component
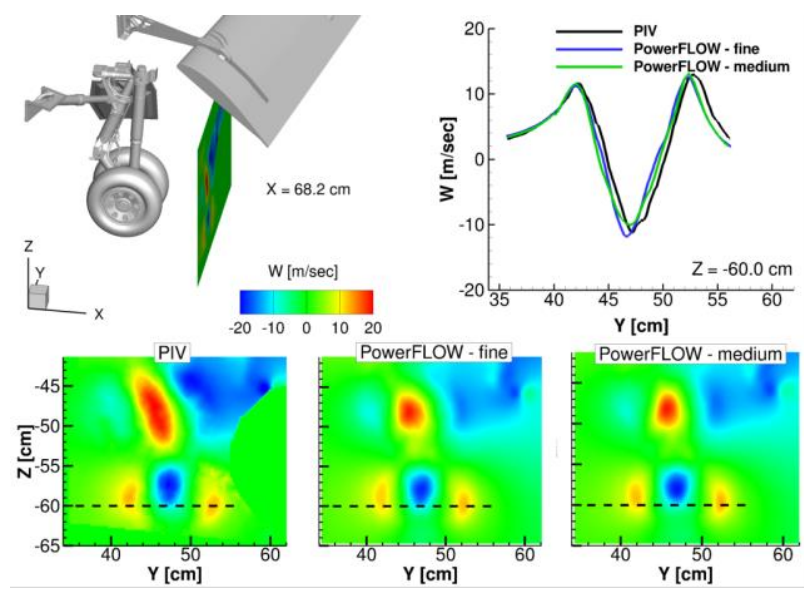

c) Mean W component

PowerFLOW - medium
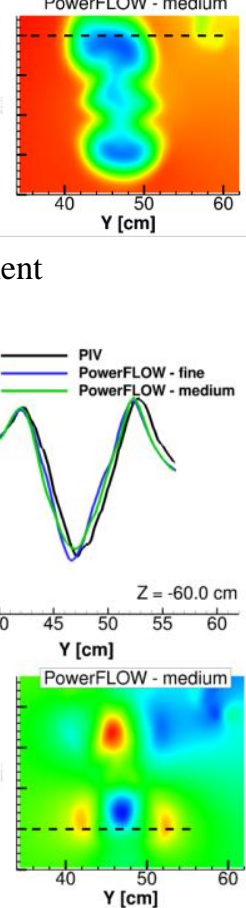
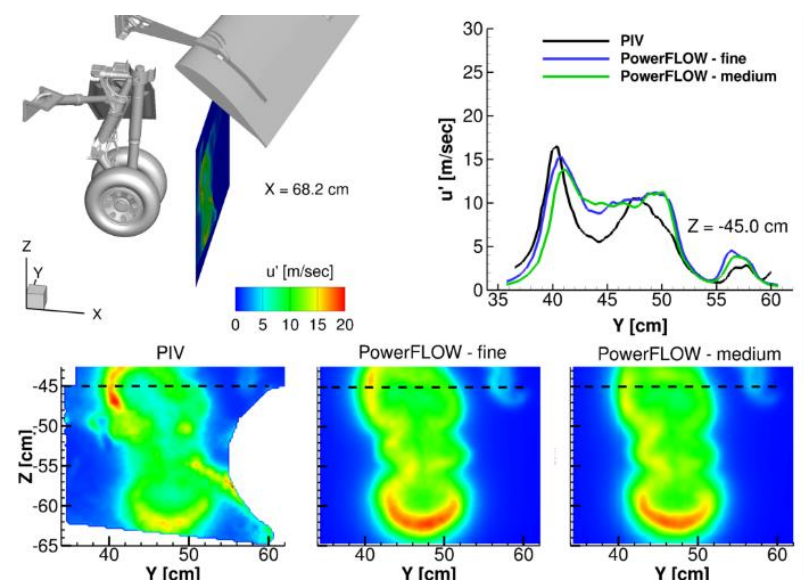

b) rms u' component
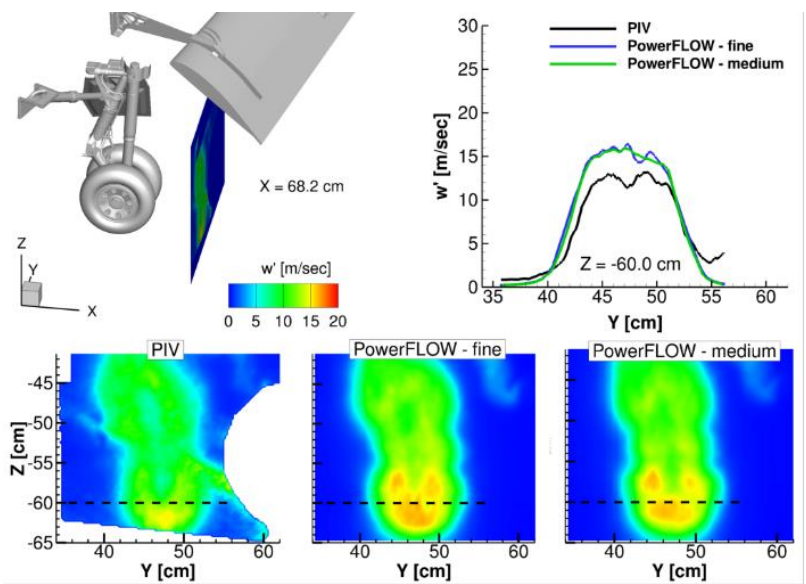

d) $\mathrm{rms} \mathrm{w}^{\prime}$ component

Figure 12. Spatial resolution effects on simulated flowfield in gear wake. $X=68.2 \mathrm{~cm}, Z=-\mathbf{4 5 . 0} \mathrm{cm}$ (a and b) and $\mathbf{- 6 0 . 0} \mathrm{cm}$ (c and d). PIV data from Run 539.

The velocity field sampled at the same streamwise location as that of Fig. 14 for the configuration with the main gear removed is shown in Fig. 15. Notice from the PIV contour in Fig. 15a that, without the gear, the tip vortex exhibits a large axial velocity excess in its core. In contrast to the results shown in Fig. 14a, comparison of the medium and fine resolution solutions of the mean velocity contours (Fig. 15a) clearly indicates that a reasonable grid independent state has not been attained. The plotted velocity profiles reinforce the visual inspection and capture the rise in vortex core velocity associated with increasing grid resolution. Contrary to the mean velocity, the simulated $u^{\prime}$ field (Fig. $15 b)$ displays better grid convergence and very good agreement with measured values. The fact that the largest differences between the simulated $\mathrm{u}^{\prime}$ profiles occur in the $54 \mathrm{~cm}<\mathrm{Y}<58 \mathrm{~cm}$ range, whereas the corresponding $\mathrm{U}$ profiles (Fig. 15a) show a grid-converged behavior over the same $\mathrm{Y}$ range, is puzzling. This unusual behavior may suggest that rather than being created by the local flow field, the fluctuations were generated at some other location and are just convecting through the measurement plane. Under this scenario, the mean and fluctuating velocities could possess a dissimilar grid convergence behavior. This peculiarity could also be linked to the length of the simulated time record used to perform the averages for the mean and fluctuating velocities. The causes of such behavior merit further scrutiny. 

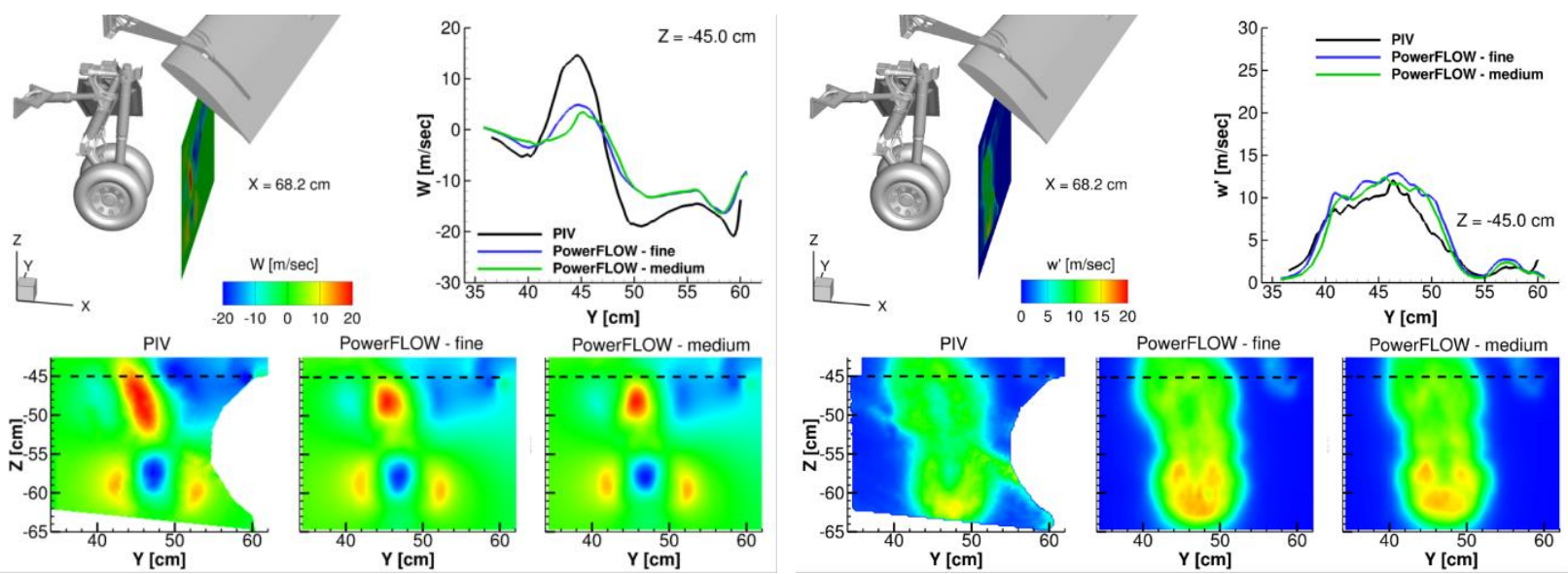

a) Mean $W$ component
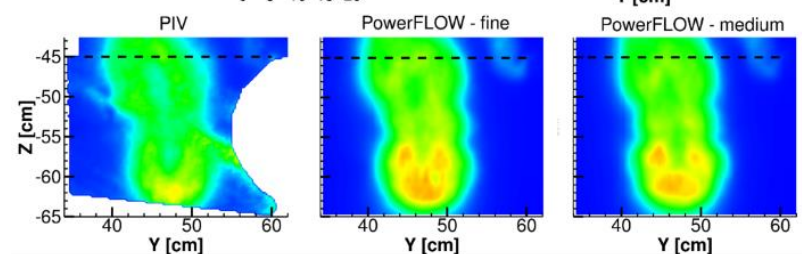

b) rms $\mathrm{w}^{\prime}$ component

Figure 13. Spatial resolution effects on simulated flowfield in gear wake $(X=68.2 \mathrm{~cm}, Z=-45.0 \mathrm{~cm})$.
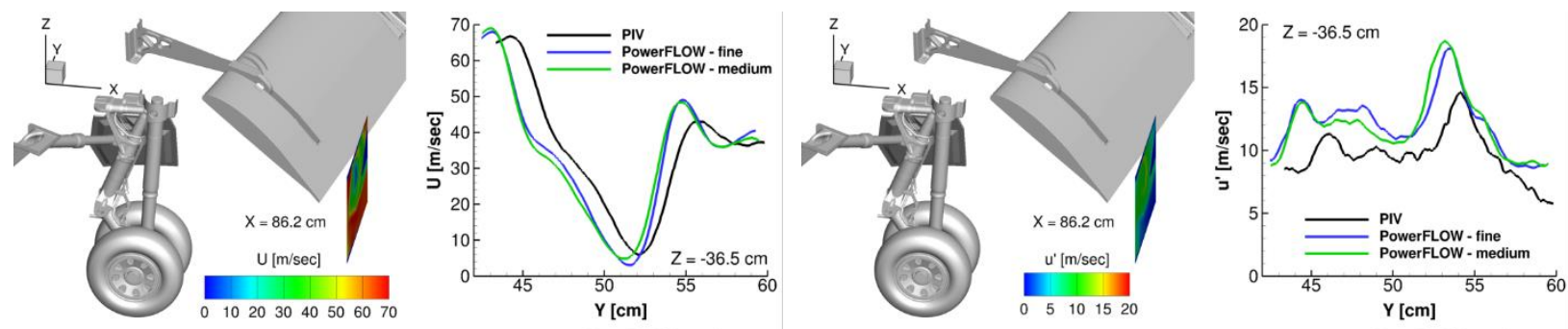

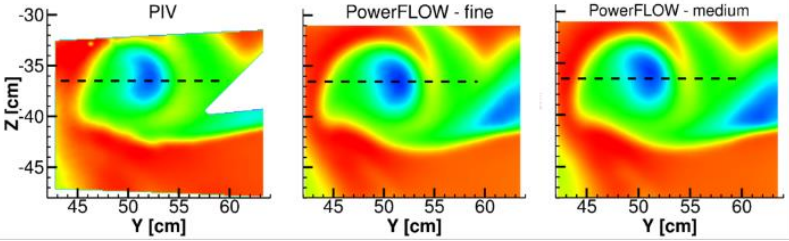

a) Mean U component

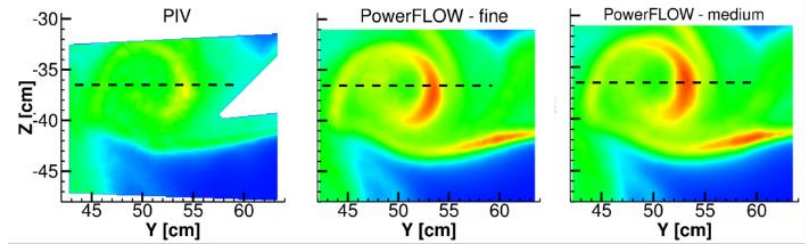

b) rms u' component

Figure 14. Spatial resolution effects on simulated flowfield in flap wake with main gear installed $(X=86.2 \mathrm{~cm}$, $\mathrm{Z}=\mathbf{- 3 6 . 5} \mathbf{c m})$.
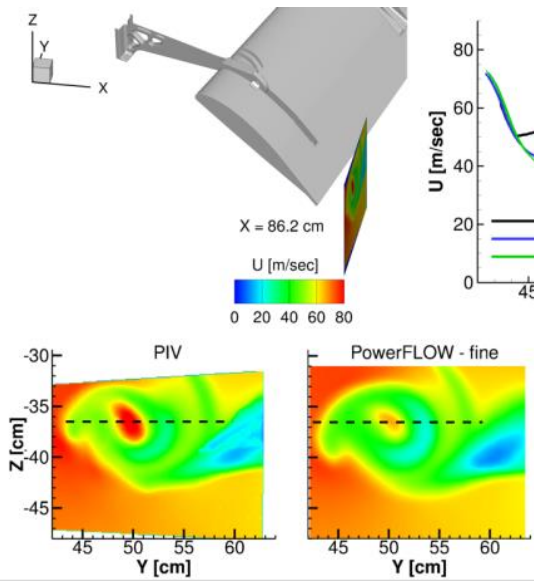

a) Mean U component
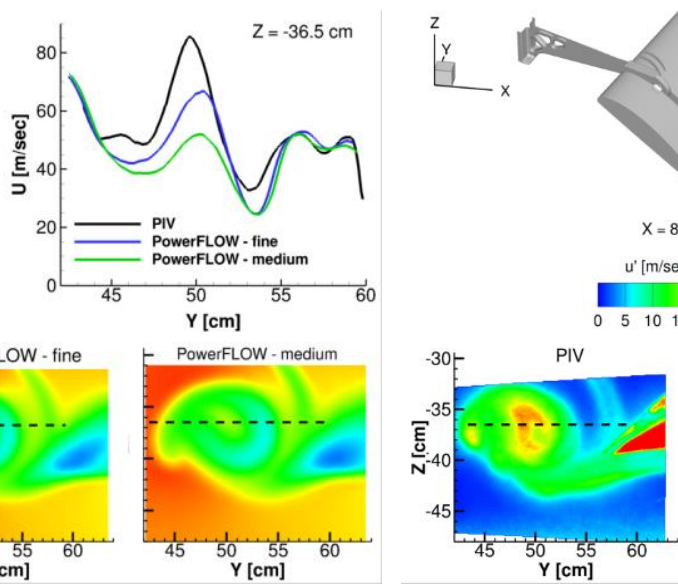
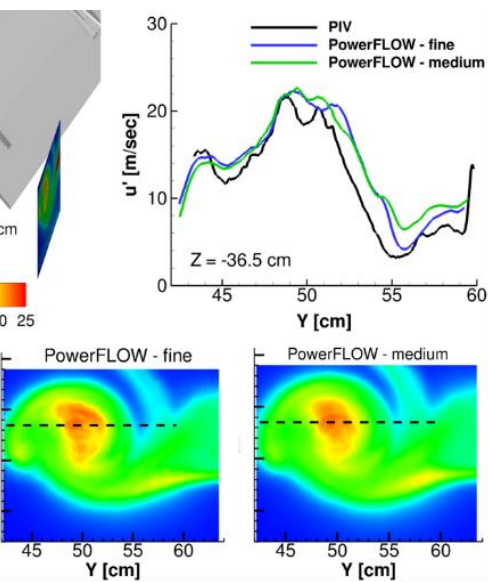

b) rms u' component

Figure 15. Spatial resolution effects on simulated flowfield in flap wake with main gear removed $(X=86.2$ $\mathrm{cm}, \mathrm{Z}=\mathbf{- 3 6 . 5} \mathrm{cm})$. 
As mentioned earlier, scrutiny of the computed mean and fluctuating velocity fields at various other locations indicated that, in general, very good solution convergence exists for the two resolutions discussed in this paper. However, the results presented in Figs. 13-15 provide ample evidence that, for highly complex and interactive flow fields such as those encountered in the gear-flap interaction zone, attainment of a grid independent, fully converged flow (in both spatial and temporal domains) is not a trivial task. Adding to this the inherent sources of uncertainty present in the PIV measurements (e.g., day to day repeatability, seeding issues, number of images taken and used for averaging, etc.), it is quite clear that obtaining good comparisons between computations and measurements is difficult at best and fraught with many pitfalls. Although the reasonably good agreement obtained between the computed and measured velocity fields is a major achievement, additional effort on both computational and experimental fronts is needed in order to realize further improvements in data quality and accuracy.

\section{B. Comparison with Experimental Measurements}

We now begin the task of comparing the solutions obtained with FUN3D and PowerFLOW® (fine resolution) with the PIV measurements at a few select locations. The upper segment of the gear wake in the vicinity of the flap inboard tip region is shown in Fig. 16 with the measurement plane situated at $X=68.2 \mathrm{~cm}$. Recall that the PIV planes in this orientation, while having a smaller FOV, possess a finer resolution compared to the lower segment of the gear wake. All mean and fluctuating velocity components are displayed. The complexity of the flow features comprising the wakes emanating from the door, struts, and upper part of the wheels is evident in the streamwise velocity contours of Figs. 16a and 16b. The door wake, which appears as the slanted, slender, and contorted structure in the middle of the contour plots (Fig. 16a) is well captured by both computations. So is the tip vortex generated at the door's bottom edge, which appears in the figures as a very small, round flow structure tethered to the lower end of the door wake. Resolution of such small flow features is a good indication of the high level of mesh resolution employed in the simulations. Inspection of other velocity components (Figs. 16c through 16f) indicates that the overall character and structure of the gear upper wake is sufficiently resolved and captured rather well. Also notice that while each of the three mean velocity fields has a distinct character, the fluctuation velocity components $\left(\mathrm{u}^{\prime}, \mathrm{v}^{\prime}\right.$, and $\left.\mathrm{w}^{\prime}\right)$ display many common features that make these contours appear very similar.

A more direct comparison between the computed and measured velocity fields is attempted in Fig. 17. The extracted profiles (along the dashed lines in Fig. 16a) are in reasonable agreement, corroborating the earlier assertion that the overall character of the upper gear wake is adequately captured. Although not negligible, the differences among the three sets of profiles generally fall within the day-to-day repeatability and resolution bounds of the PIV measurements, uncertainties associated with the spatial resolution and the length of solution record (used to perform averages) of the computed flow field, and the differences in the experimental (open-jet) and computational (free-air) setups.

The contour plots for the full gear wake at $X=68.2 \mathrm{~cm}$ are displayed in Fig. 18. As discussed in section II.B.a, the upper segment of this plane coincides with the higher resolution, smaller FOV plane shown in Fig. 16. As seen in the streamwise velocity field (Fig. 18a), the gear main wake at this X location appears in the general shape of a dumbbell with the lowest velocities residing at the core of the two main lobes. The isolated structure adjacent to the wake in the center left section of the PIV contour is an anomaly caused by laser light reflected off the landing gear wheel. The same anomalous region can be observed in the V contours (Fig. 18b), where it masks part of the highest positive velocity region. The upper lobe, which contains the wakes originating from the knee section of the front strut (see Fig. $2 \mathrm{~b}$ ) and the top portion of the wheels, is the largest structure. The lower lobe contains the wakes emanating from the two wheels and the structures residing between them (e.g., axle, brake calipers and hydraulic lines, etc.). The mean flow fields produced by the two simulations (Figs. 18a, 18c, and 18e) look remarkably similar to the measured field. Good agreement is also observed for the fluctuation fields (Fig. 18b, 18d, and 18f). The $u^{\prime}$ contour plot suggests that higher amplitude streamwise fluctuations occur in the periphery of the gear wake along the side opposite to the door location. In contrast, the $v^{\prime}$ contour indicates that the stronger fluctuations reside in the interior of the wake, mainly in the region connecting the two lobes. For the $\mathrm{w}^{\prime}$ component, the dominant fluctuations occur inside the wake's lower lobe. Qualitatively speaking, both simulations have captured the prominent features of the fluctuating velocity field; thus, the results and subsequent comparison with measured data are reasonably good.

Comparisons of the mean and fluctuating velocity profiles are provided in Fig. 19. The sample profiles were extracted along the $\mathrm{Y}$ and $\mathrm{Z}$ coordinates at three select positions: $\mathrm{Z}=-60.0 \mathrm{~cm}$ and $-40.0 \mathrm{~cm}$, and $\mathrm{Y}=46.0 \mathrm{~cm}$ (dashed lines in Fig. 18a). As gleaned from observation of the contours in Fig. 18, the simulated mean velocity profiles compare rather well to each other and to the measured profiles. Achieving this level of agreement is quite encouraging, given the host of computational and experimental factors that could affect data accuracy. Inspection of the velocity profiles depicted in Fig. 19a indicates that the lower portion (lobe) of the gear wake is highly symmetric. The streamwise mean velocity component (U) shows a rather large deficit of about $50 \mathrm{~m} / \mathrm{sec}$, indicating that wake recovery has 
not occurred at this station. The profiles through the upper portion (lobe) of the wake depict a noticeable degree of asymmetry due to the presence of the door (Fig. 19b). The asymmetry extends to the fluctuating velocity field, with u' being the most affected component.

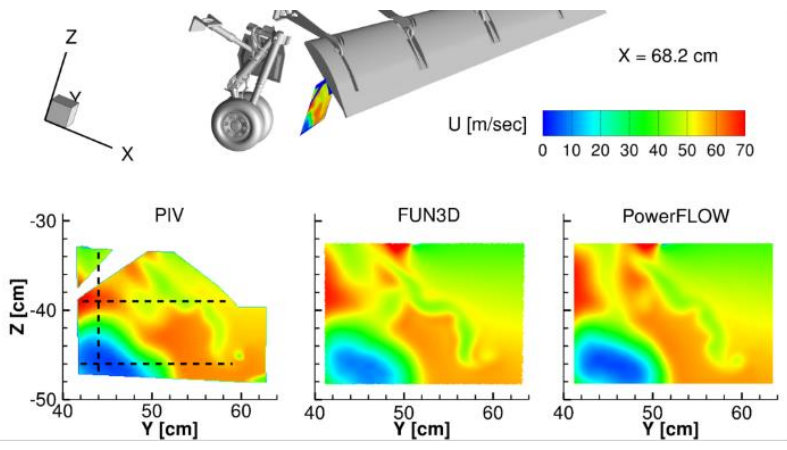

a) Mean U component
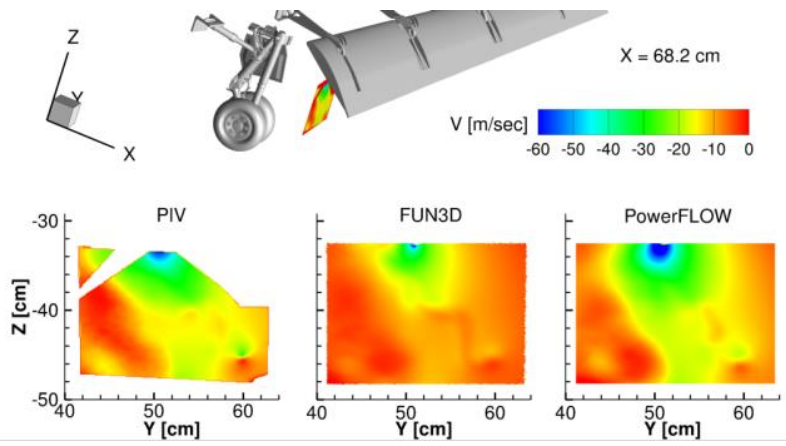

c) Mean V component
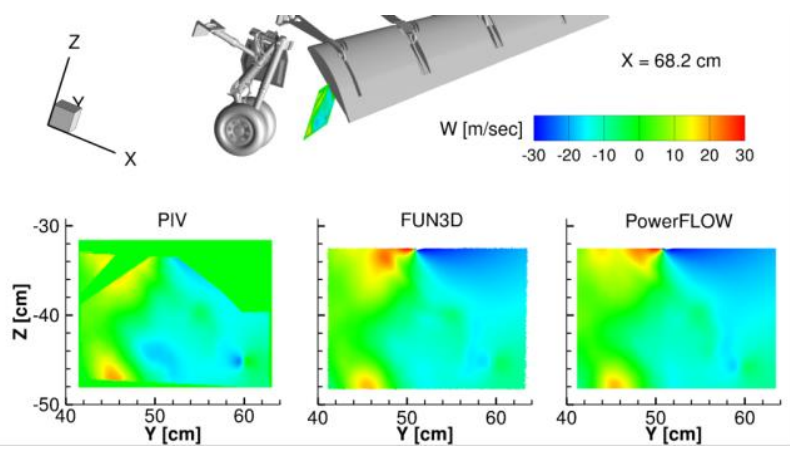

e) Mean W component
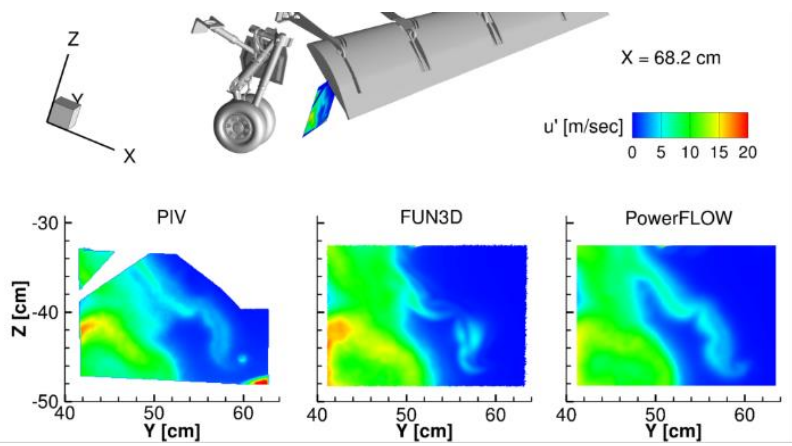

b) rms u' component
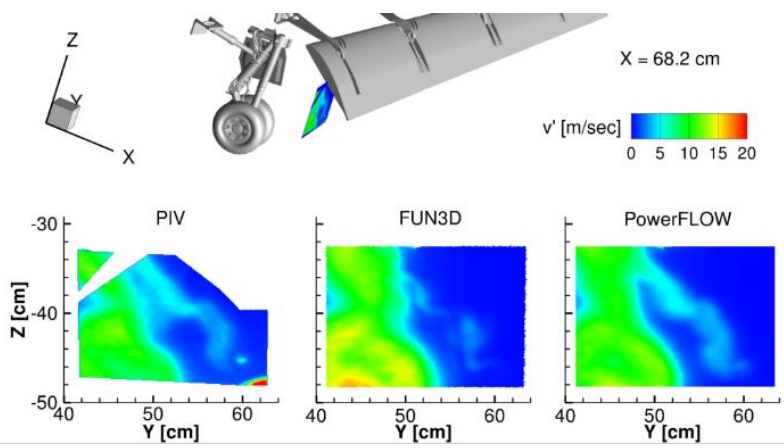

d) rms v' component
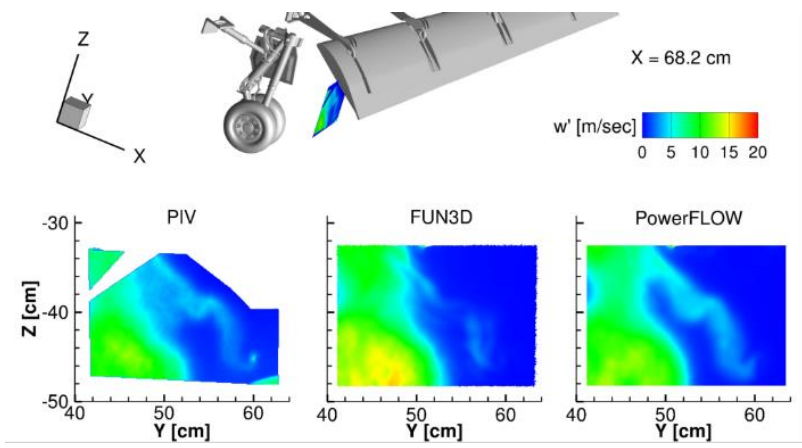

f) rms w' component

Figure 16. Mean and fluctuating velocity contours for upper gear wake $(X=68.2 \mathrm{~cm})$

Velocity contours at one of the 2D-PIV measurement planes are shown in Fig. 20. This plane, situated at approximately mid-height of the gear strut $(\mathrm{Z}=-35.5 \mathrm{~cm})$, captures the streamwise development of the wake in the region between the gear and the inboard flap tip. Examination of the mean streamwise velocity (Fig. 20a) at this location indicates that the wakes from the main gear door and strut are distinct and separated by a high speed flow region. The door produces a much narrower wake relative to the strut. Also notice that the entire wake field is deflected sideways, drawn towards the low pressure region near the flap tip. The resulting spanwise flow is captured in the $\mathrm{V}$ contours (Fig. 20c), where higher negative velocities as the wake approaches the flap side edge are shown. Contours for the $u^{\prime}$ and v'components depict very similar behavior; as expected, the highest rms values occur at the outer edges of the strut wake presumably due to the separated shear layers and vortex shedding process. 
The corresponding velocity contours from the FUN3D and PowerFLOW ${ }^{\circledR}$ simulations produce a similar qualitative picture of the flowfield, capturing most of the finer details associated with wake development at this location. A quantitative comparison of the computed and measured velocity fields, in the form of mean and fluctuation velocity profiles along the $\mathrm{Y}$ axis, is presented in Fig. 21. The profiles were extracted at the two constant $\mathrm{X}$ stations highlighted by the vertical dashed lines in the PIV contour image of Fig. 20. The streamwise velocity profiles nearest to the gear (Fig. 21a) clearly show the velocity deficits of the wakes associated with the door and strut. The highest fluctuation levels occur in the wake of the strut, which shows a double peak profile typical of symmetrical bluff bodies. Farther downstream at the $X=63 \mathrm{~cm}$ station, the deficits in the mean streamwise velocity are diminished considerably while the magnitude of the fluctuation levels is mostly maintained. Both simulations produced profiles that capture the shape and magnitude of measured data. Based on visual inspection it appears that, in general, the profiles extracted from the PowerFLOW® solution show better agreement with the measured data. This is not entirely surprising, since the mesh resolution within the gear-flap interaction region was finer than that of the mesh used with FUN3D.
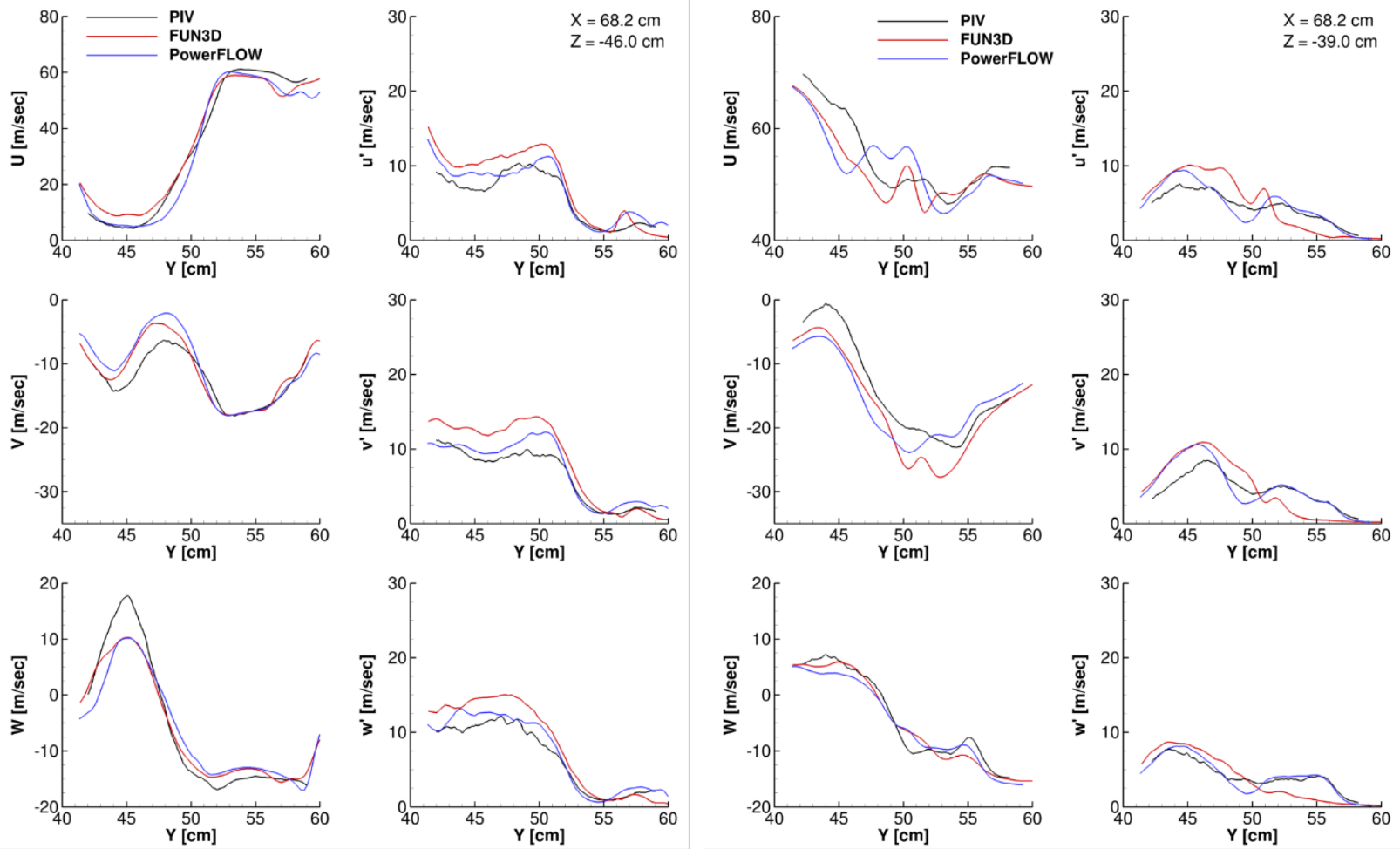

a) $\mathrm{Z}=-46.0 \mathrm{~cm}$ station

b) $\mathrm{Z}=-39.0 \mathrm{~cm}$ station

Figure 17. Mean and fluctuating velocity profiles for upper gear wake $(X=68.2 \mathrm{~cm})$ 

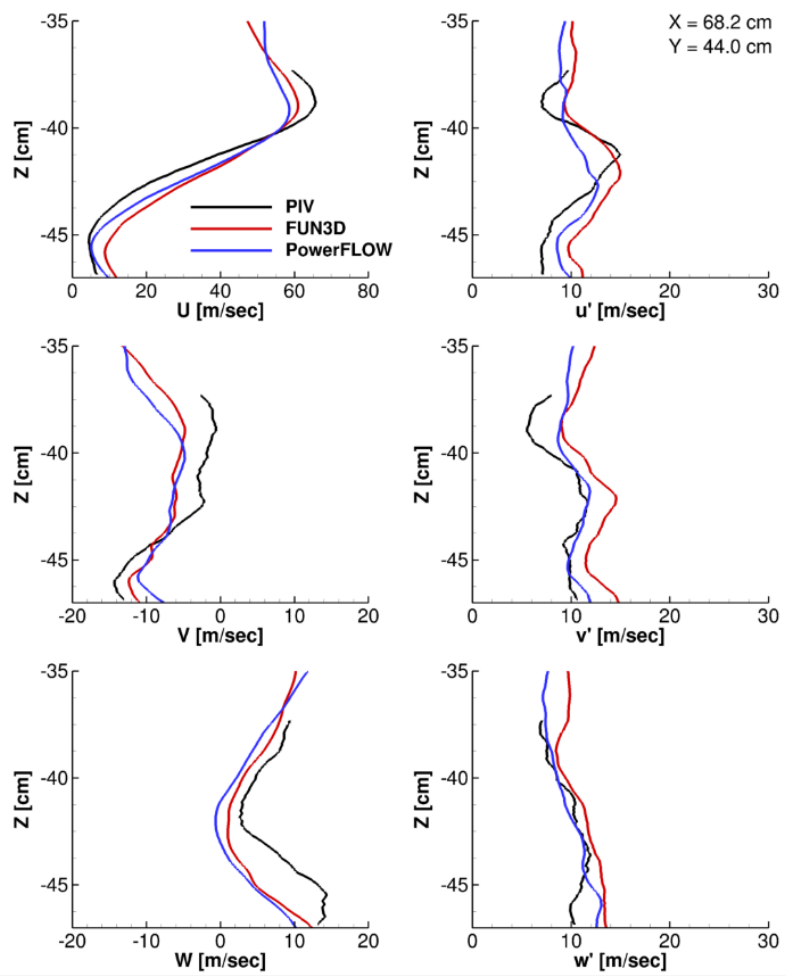

c) $\mathrm{Y}=44.0 \mathrm{~cm}$ station

Figure 17. Concluded.

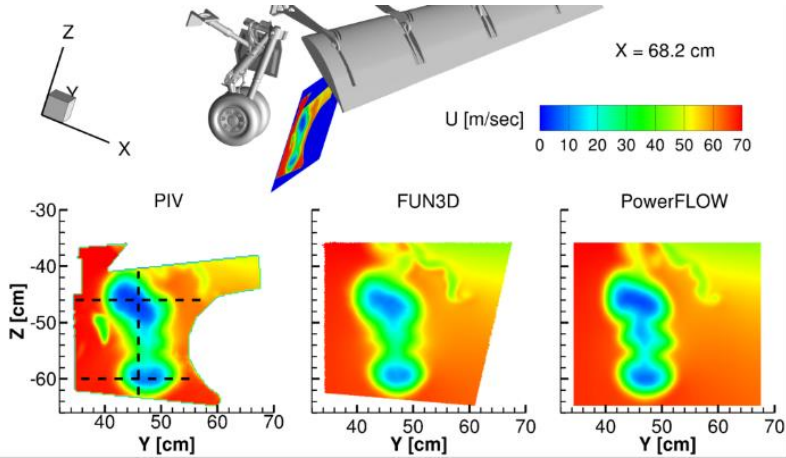

a) Mean U component
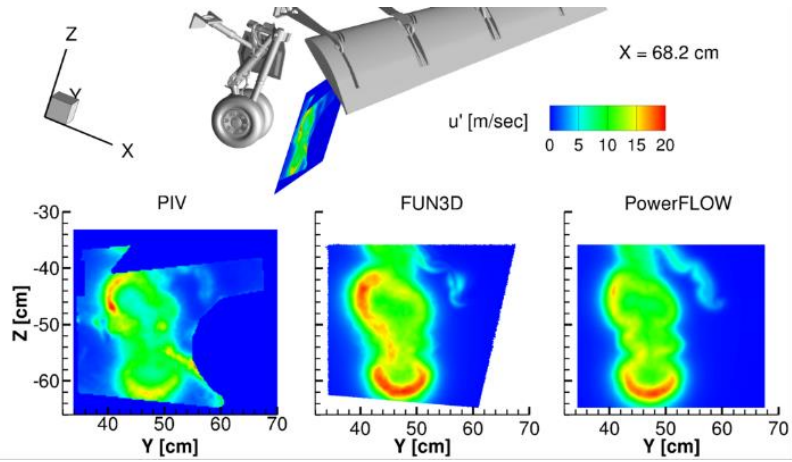

b) rms u' component

Figure 18. Mean and fluctuating velocity contours for full gear wake $(X=68.2 \mathrm{~cm})$ 

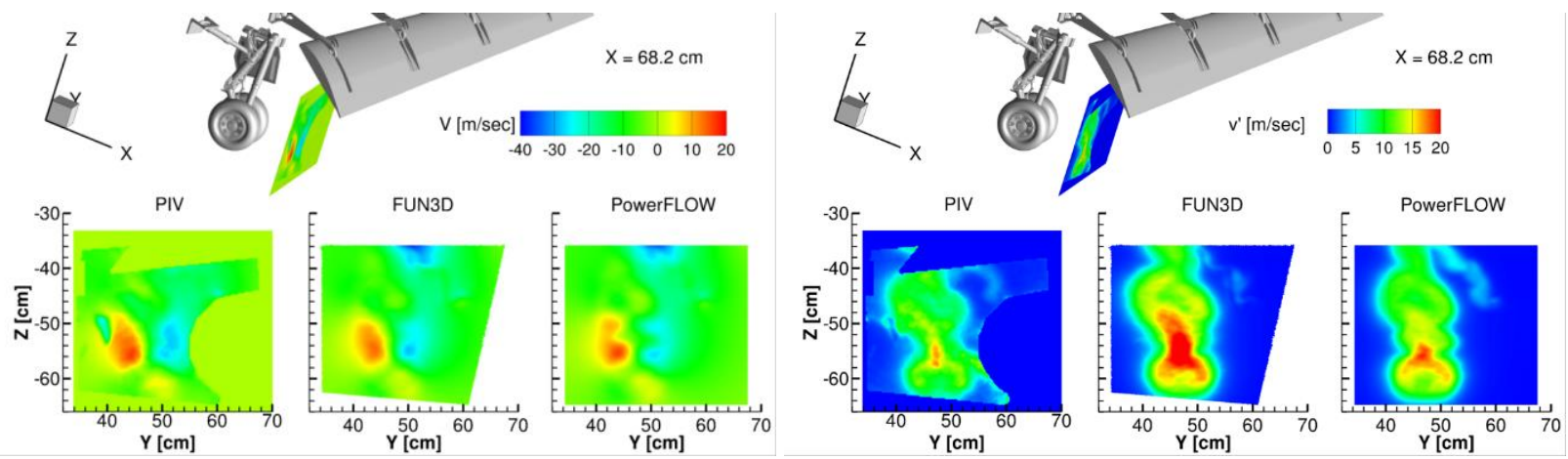

c) Mean V component

d) rms v' component
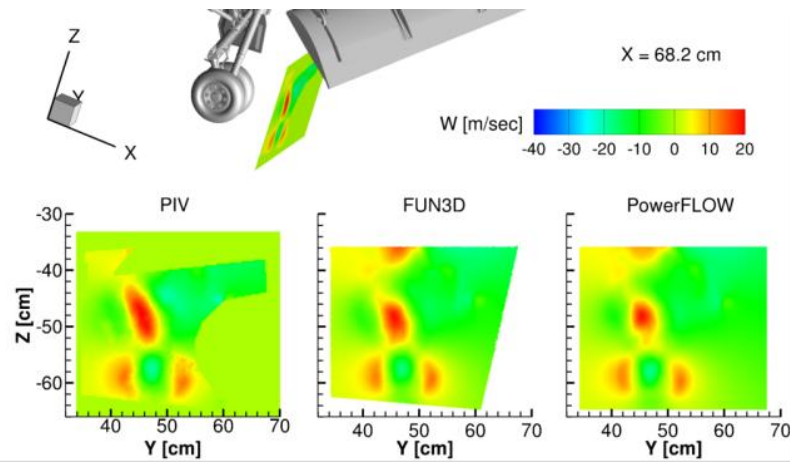

e) Mean W component
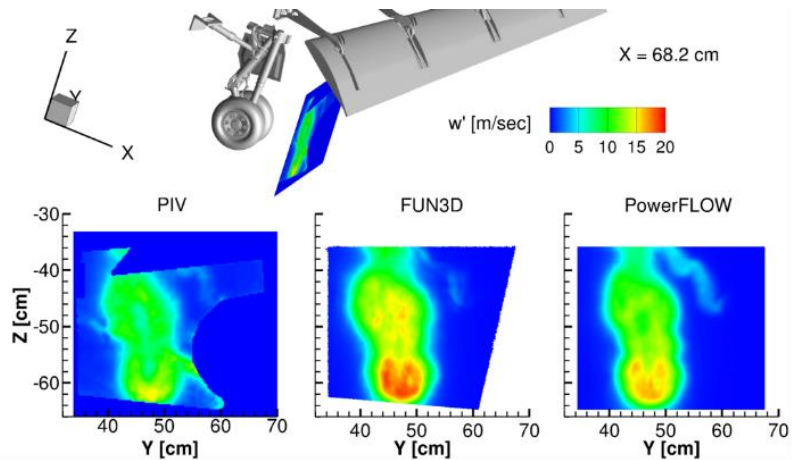

f) rms w' component

Figure 18. Concluded.
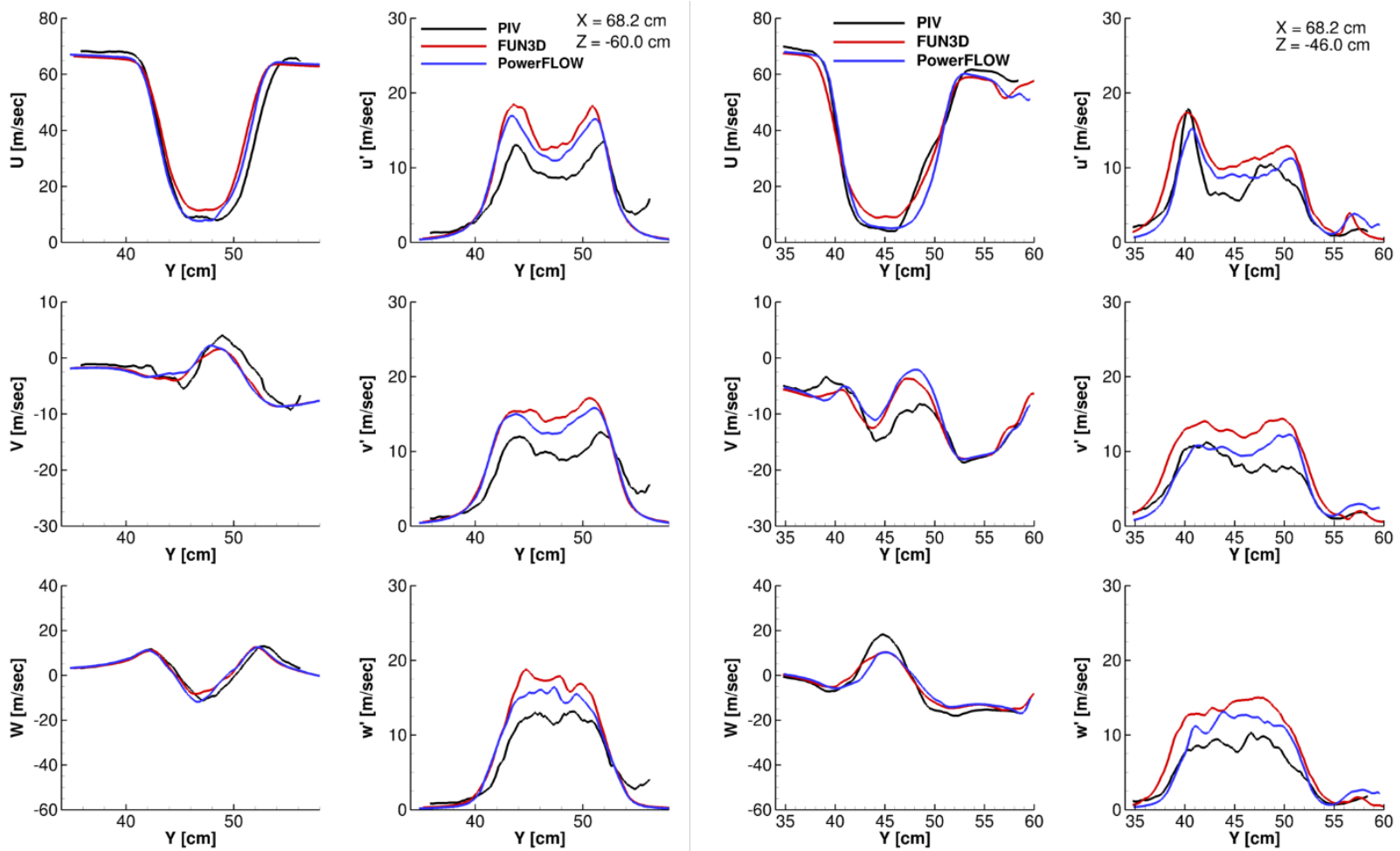

a) $\mathrm{Z}=-60.0 \mathrm{~cm}$ station

b) $\mathrm{Z}=-46.0 \mathrm{~cm}$ station

Figure 19. Mean and fluctuating velocity profiles for full gear wake $(X=68.2 \mathrm{~cm})$ 

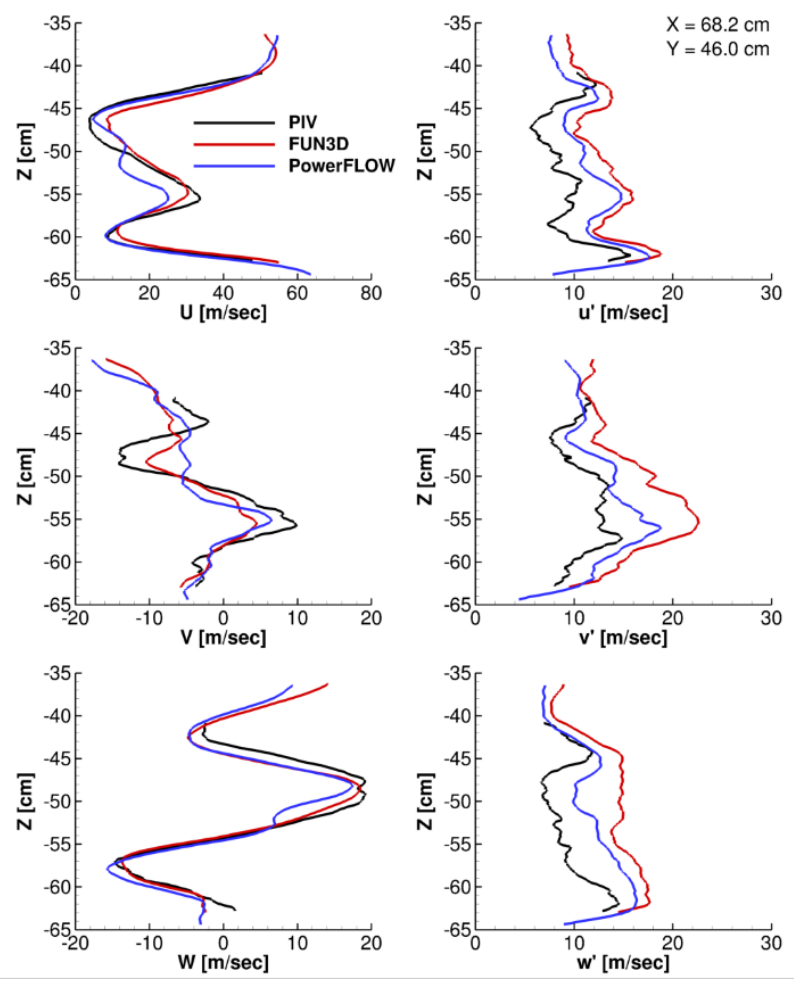

c) $\mathrm{Y}=46.0 \mathrm{~cm}$ station

Figure 19. Concluded.

Contour plots from one of the SPIV measurement planes downstream of the flap trailing edge at $X=86.2 \mathrm{~cm}$ are shown in Fig. 22. Velocity contours for both gear on and gear off configurations are presented. In general, very good agreement between the measured and the two computed mean velocity fields is observed. Note, again, that contours from PowerFLOW® simulations show closer agreement with the PIV data due to better resolution in the flap tip region than FUN3D computations. Looking at the mean streanwise velocity contours of Fig. 22a, observe that there is a significant difference in the magnitude of axial velocities encountered within the core as well as in the general shape of the flap tip vortex for the two model configurations shown. With the gear on (top row plots), the tip vortex displays significant axial velocity deficits in its core approaching a zero value. In contrast, with the gear removed (bottom row plots), the vortex shows a substantial increase in its core velocities with values reaching or even exceding freestream levels. The V and $\mathrm{W}$ components of the mean velocity (Figs. 22c and 22e) show larger magnitudes for the gear off configuration, indicating that higher rotational velocities and lower static pressures exist in the vortex core. Examination of the $\mathrm{u}^{\prime}, \mathrm{v}^{\prime}$, and $\mathrm{w}^{\prime}$ contours reveals that all three components possess similar characteristics and magnitude levels. For the gear on configuration, the most intense fluctuations occur in a ring surrounding the vortex core. Recall from Fig. 22a that the mean streamwise velocity for this configuration is nearly zero at this X station. The presence of near zero velocities indicates that either a vortex breakdown has already occurred or is about to occur, causing a rapid expansion of the core that displaces the fluctuations to the periphery of the vortex. In contrast, the fluctuation contours for the gear off configuration show that the most intense perturbations are concentrated within the vortex core. In general, the FUN3D contours hint at overprediction of fluctuation magnitudes with the PowerFLOW® results in better agreement with the PIV measurements.

Mean and fluctuation velocities extracted along $\mathrm{Z}=-37.0 \mathrm{~cm}$ and $\mathrm{Y}=51 \mathrm{~cm}$ (dashed lines in the PIV contours) are displayed in Fig. 23. The large deficit in the axial velocity for the gear on configuration is clearly visible in Fig. 23a. Gear off results (bottom rows in Fig. 23) indicate that all three fluctuating velocity components have higher amplitudes relative to the configuration with the main gear deployed. 

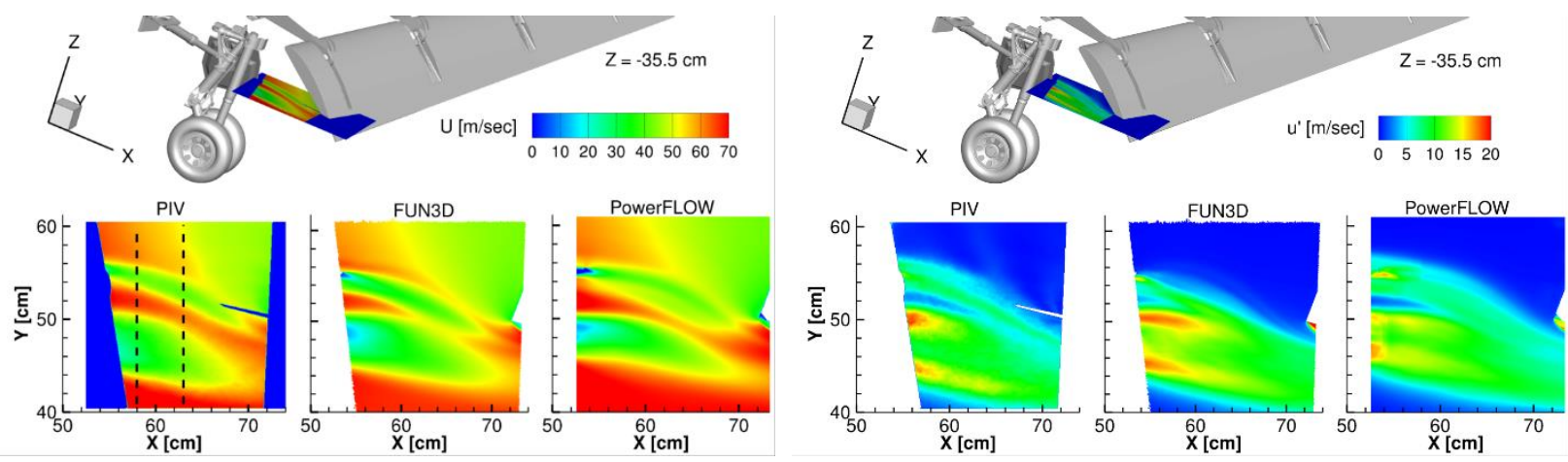

a) Mean U field

b) $\mathrm{rms} \mathrm{u}^{\prime}$ field
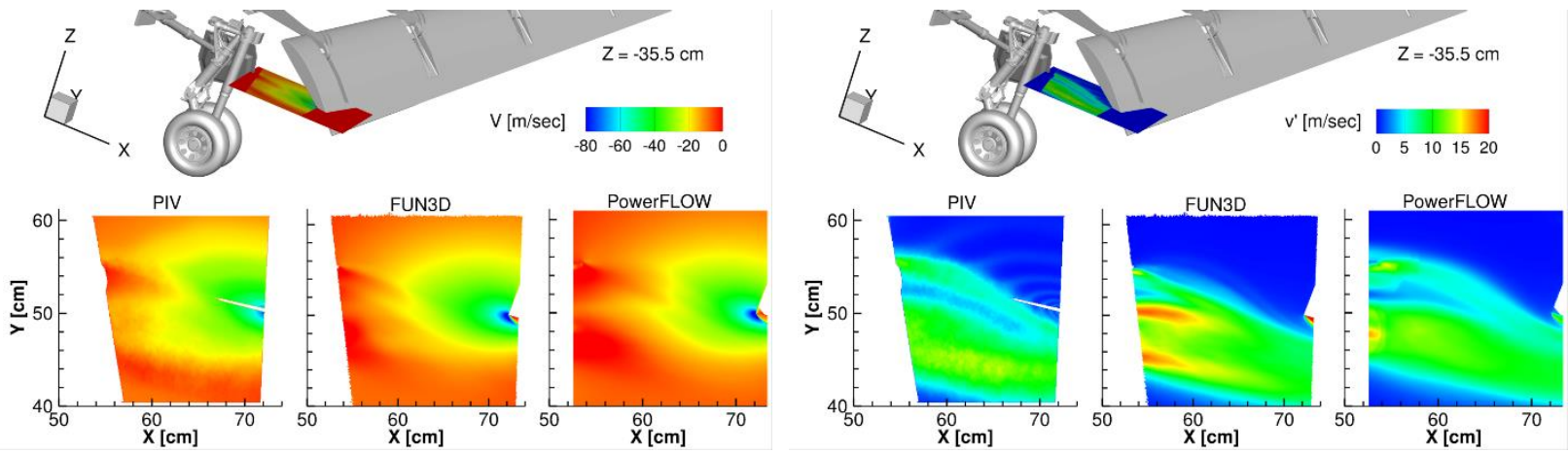

c) Mean $V$ field

d) rms $v^{\prime}$ field

Figure 20. Streamwise development of main landing gear wake $(Z=-35.5 \mathrm{~cm})$
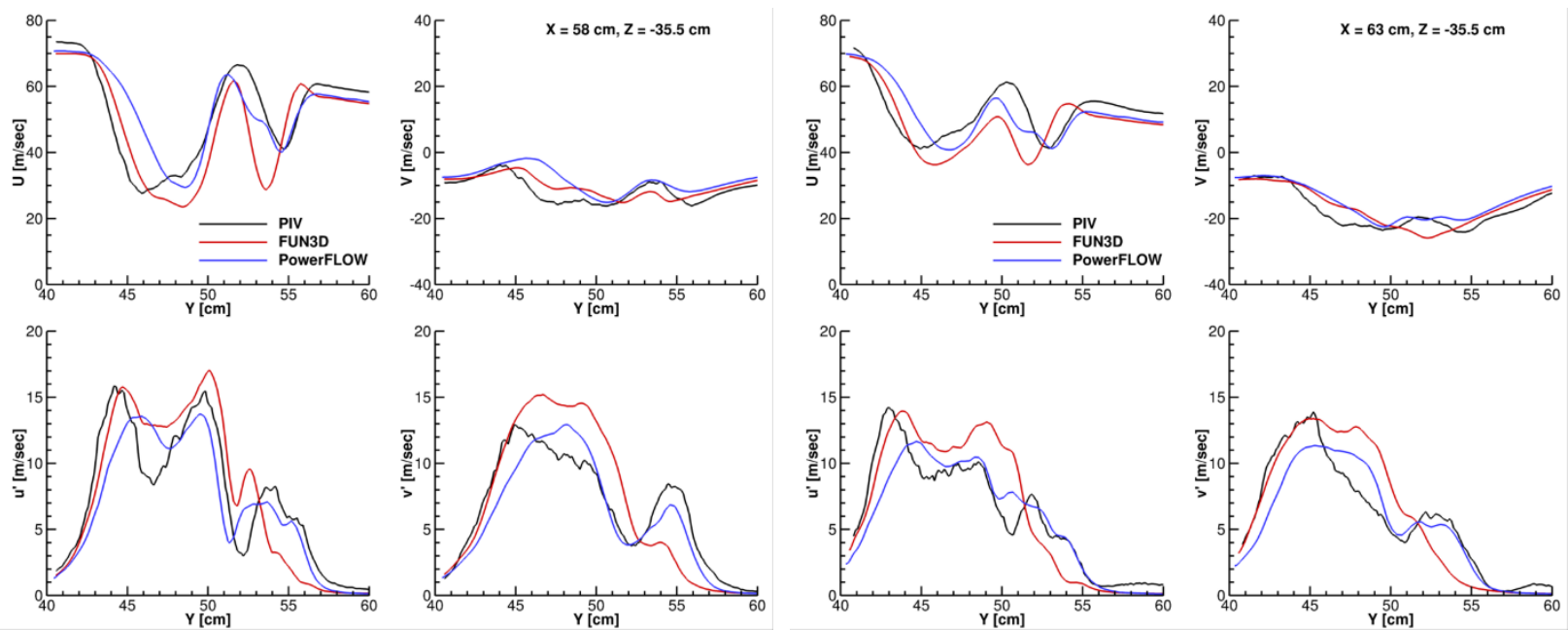

a) $\mathrm{X}=58 \mathrm{~cm}$ station

b) $\mathrm{X}=63 \mathrm{~cm}$ station

Figure 21. Mean and fluctuation velocity profiles at two streamwise stations $(Z=-35.5 \mathrm{~cm})$ 

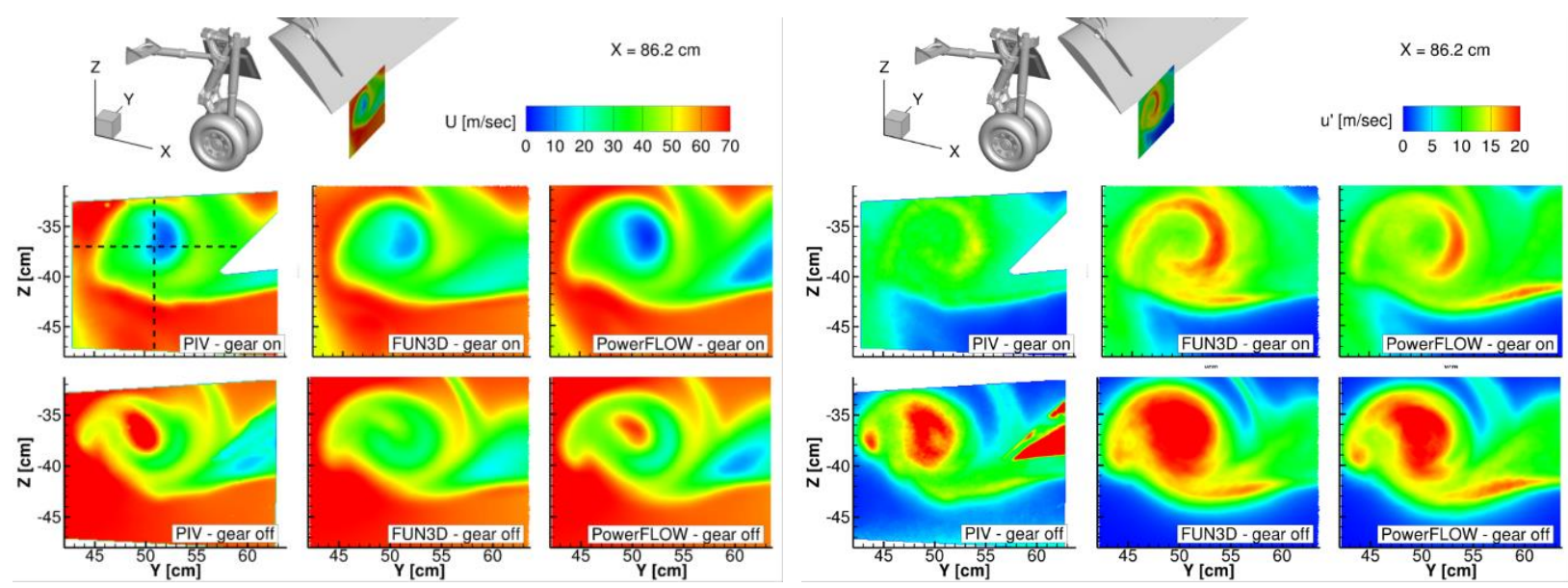

a) Mean U component
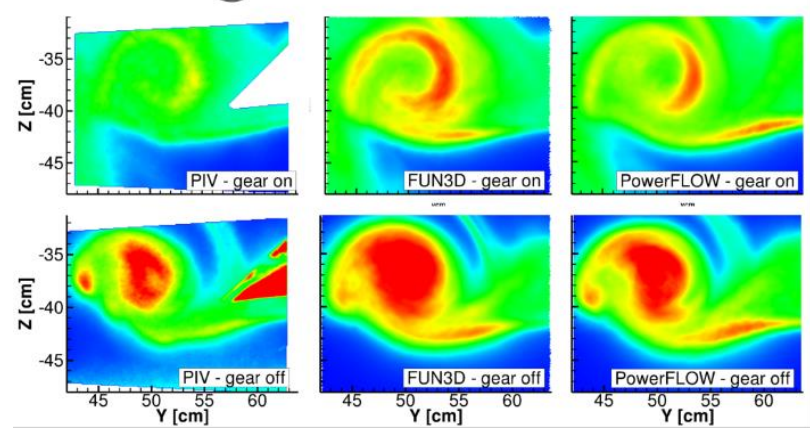

b) rms u' component
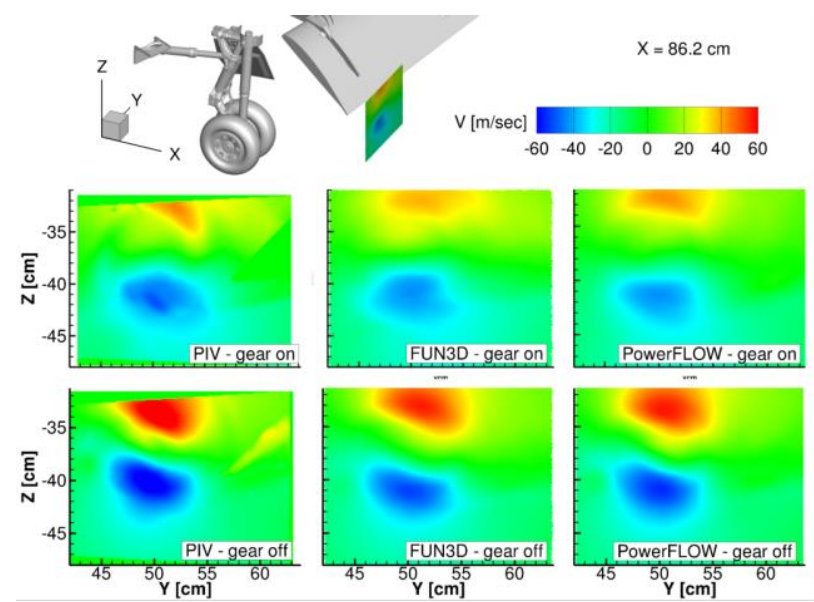

c) Mean V component
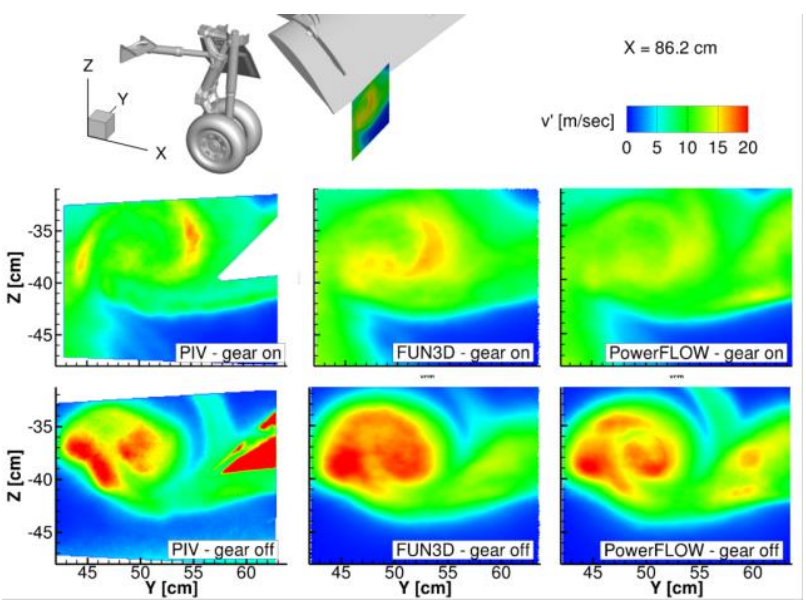

d) rms $v^{\prime}$ component
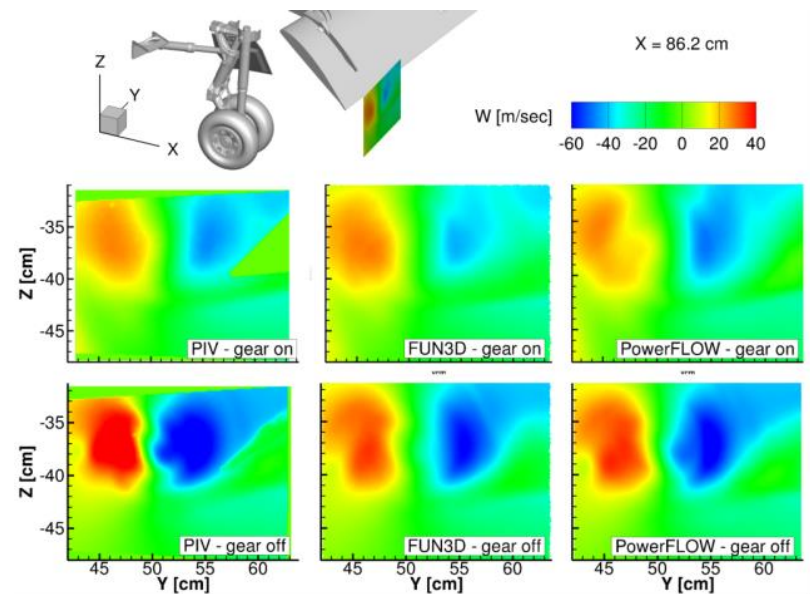

e) Mean W component
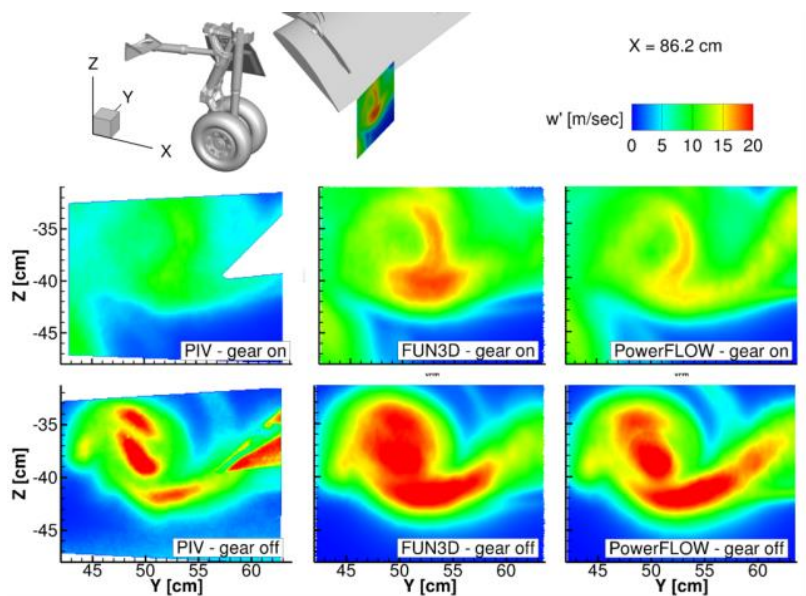

f) rms w' component

Figure 22. Comparison of velocity contours downstream of flap trailing edge showing the tip vortex $(\mathrm{X}=\mathbf{8 6 . 2} \mathrm{cm})$. 

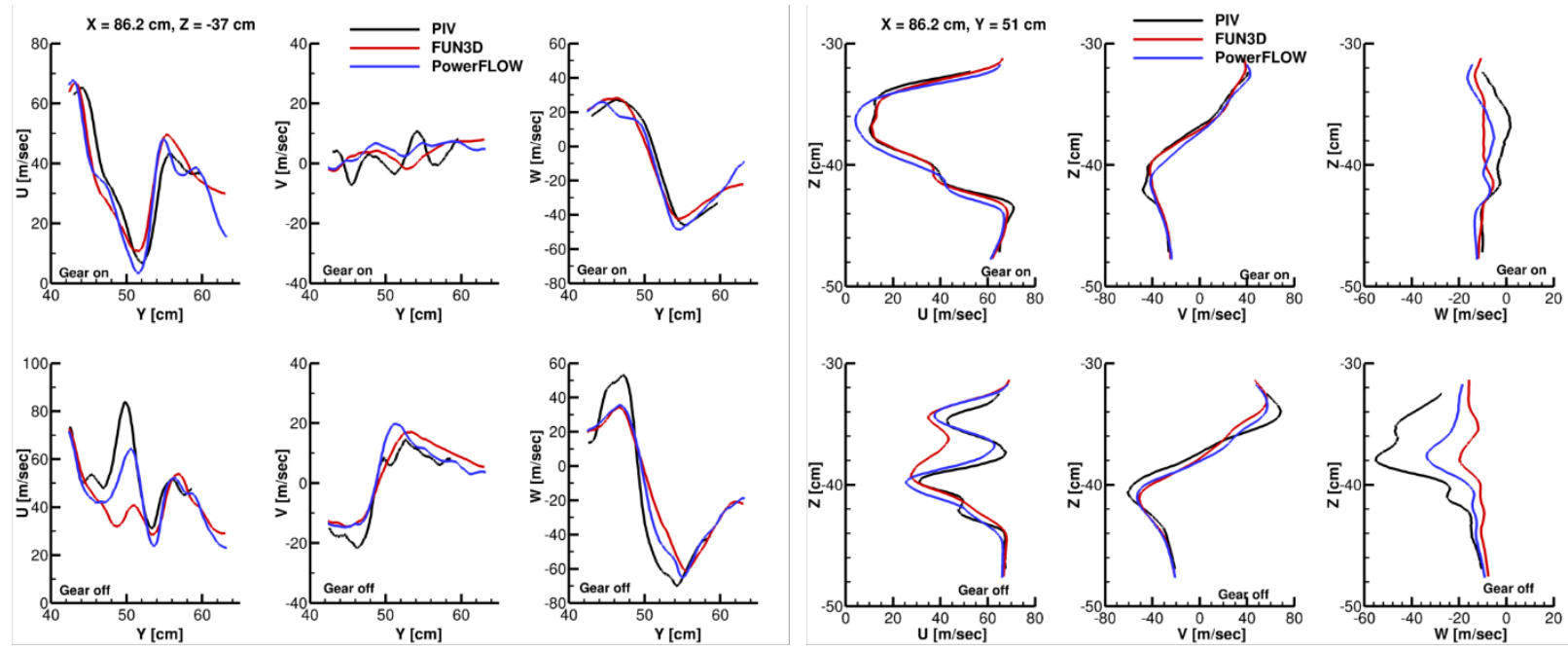

a) Mean velocities along $\mathrm{Y}$ and $\mathrm{Z}$ coordinates; top row gear on and bottom row gear off
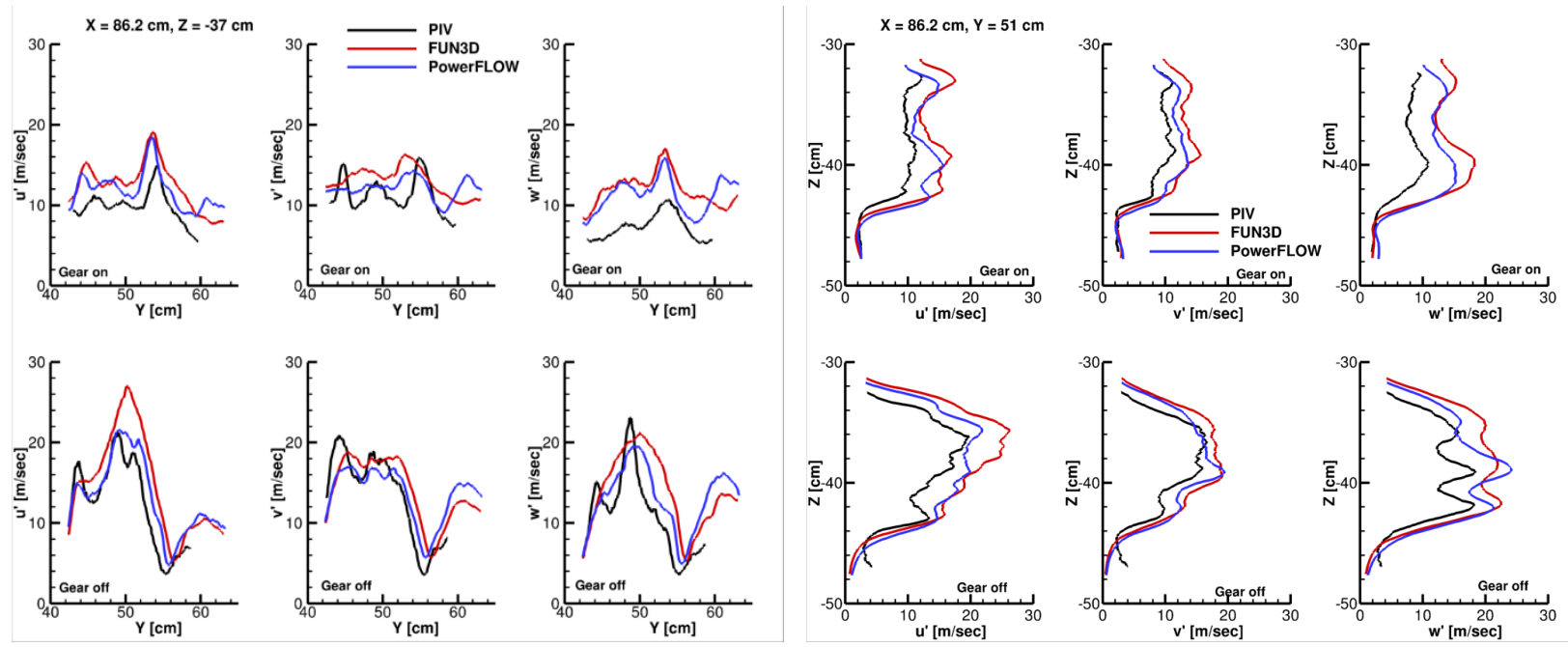

b) Fluctuation velocities along $\mathrm{Y}$ and $\mathrm{Z}$ coordinates; top row gear on and bottom row gear off

Figure 23. Comparison of mean and fluctuation velocity profiles for gear on and gear off configurations $(\mathrm{X}=86.2 \mathrm{~cm})$

\section{Concluding Remarks}

Simulated and measured velocity fields for a realistic gear-flap flow interaction zone were assessed in this paper. The computational results were obtained with NASA's FUN3D and Exa's PowerFLOW® solvers for a high-fidelity, $18 \%$ scale semi-span model of a Gulfstream aircraft in landing configuration with and without main landing gear. 2DPIV and SPIV techniques were used to sample the flow within the gear-flap interaction zone during wind tunnel tests of the same aircraft model. Limited spatial resolution and day-to-day variability effects on the measured velocity field were presented and discussed. Although not negligible, these factors were deemed to not affect the main features of the gear-flap flow interaction. Mesh refinement effects on the accuracy of the flowfield generated using PowerFLOW® were highlighted via comparisons between medium and fine resolution results. Reasonably good agreement between the two resolutions was observed at the majority of the locations examined, indicating that the computed mean and fluctuating velocity fields were approaching a near mesh-independent state. The effects of mesh refinement on the FUN3D computed flow was not addressed because the simulation was executed using the finest grid that could be constructed within the scope of this study.

A detailed comparison among the two computational data sets and PIV data sampled at a few select planes was provided. It was shown that both computed flowfields capture the relevant characteristics of the landing gear wake 
and gear-flap interaction zone rather well. The global features and downstream evolution of the wakes associated with the gear door, struts, and wheels were predicted with reasonable accuracy. A more direct comparison among computed and measured velocity fields was provided using profiles of the mean and fluctuating velocity components extracted at various stations. Examination of the velocity contours and profiles at the measurement plane slightly downstream of the flap trailing edge showed marked differences in the tip vortex character between the gear on and gear off configurations. With the gear deployed, the vortex was larger with lower rotational speeds and a substantial axial velocity deficit having minimum levels approaching zero in its core. Accordingly, it was shown that the velocity fluctuations of largest amplitude were concentrated in the periphery of the vortex core. With the gear removed, the vortex was found to be somewhat tighter with higher rotational speeds and velocity surplus in its core. For this configuration, the larger amplitude fluctuations were found to be concentrated inside the core. Both computational data sets were shown to capture the gear effects on the flap tip vortex reasonably well.

The present comparative study clearly indicates that good agreement among simulated and measured mean and fluctuating velocities was obtained, with the PowerFLOW® results providing somewhat closer agreement possibly due to the finer grid resolution employed. Given the errors potentially introduced in the measured data by the PIV system limitations/operations, a rigorous examination of the accuracy of the computed flow fields was beyond the scope of the present study. However, the partial validation of simulated off-surface volumetric velocity data included here, in conjunction with an earlier extensive validation of the on-surface steady and unsteady surface pressures, indicate that the FUN3D and PowerFLOW $®$ data sets can be utilized with confidence to extract information on the intricacies associated with gear-flap flow interaction.

\section{Acknowledgments}

This work was supported by the Environmentally Responsible Aviation (ERA) project under the Integrated System Research Program (ISRP) of NASA. The authors would like to thank Dr. Ehab Fares of Exa Corporation for providing the PowerFLOW simulation results.

\section{References}

${ }^{1}$ Dobrzynski, W., “Almost 40 Years of Airframe Noise Research: What Did We Achieve,” J. Aircraft, Vol. 47, No. 2, MarchApril 2010

${ }^{2}$ Khorrami, M. R. and Mineck, R. E., “Towards Full Aircraft Airframe Noise Prediction: Detached Eddy Simulations,” AIAA Paper 2014-2480, 2014.

${ }^{3}$ Khorrami, M. R., Fares, E., and Casalino, D., "Towards Full-Aircraft Airframe Noise Prediction: Lattice-Boltzmann Simulations," AIAA Paper 2014-2481, 2014.

${ }^{4}$ Khorrami, M. R., Hannon, J. A., Neuhart, D. H., Markowski, G. A., and Van de Ven, T., "Aeroacoustic Studies of a HighFidelity Aircraft Model: Part 1-Steady Aerodynamic Measurements," AIAA Paper 2012-2233, 2012.

${ }^{5}$ Khorrami, M. R. and Neuhart, D. H, “Aeroacoustic Studies of a High-Fidelity Aircraft Model: Part 2-Unsteady Surface Pressures," AIAA Paper 2012-2234, 2012.

${ }^{6}$ Neuhart, D., Hannon, J., and Khorrami, M. R., "Aerodynamic Measurements of a Gulfstream Aircraft Model with and without Noise Reduction Concepts," AIAA Paper 2014-2477, 2014.

${ }^{7}$ Yao, C.-S., Jenkins, L. N, Bartram, S. M., Harris, J., Khorrami, M. R., and Mace, W. D., "Flow-Field Investigation of GearFlap Interaction on a Gulfstream Aircraft Model," AIAA Paper 2014-2479, 2014.

${ }^{8}$ Biedron, R. T., Derlaga, J. M., Gnoffo, P. A., Hammond, D. P., Jones, W. T., Kleb, B., Lee-Rausch, E. M., Nielsen, E. J., Park, M. A., Rumsey, C. L., Thomas, J. L., and Wood, W. A., "FUN3D Manual: 12.4,” NASA TM 2014-218179, March 2014.

${ }^{9}$ Spalart, P. R. and Allmaras, S. R., “A One-Equation Turbulence Model for Aerodynamics Flows,” Recherche Aerospatiale, No. 1,, pp. 5-21, 1994.

${ }^{10}$ Fares, E. and Noelting, S., "Unsteady Flow Simulation of a High-Lift Configuration using a Lattice-Boltzmann Approach," AIAA Paper 2011-0869, 2011.

${ }^{11}$ Yakhot, V. and Orszag, S. A., "Renormalization Group Analysis of Turbulence. I. Basic Theory," J. Sci. Comput., Vol. 1, No. 2, pp. 3-51, 1986. 